TRANSACTIONS OF THE

AMERICAN MATHEMATICAL SOCIETY

Volume 362, Number 4, April 2010, Pages 2089-2113

S 0002-9947(09)04867-3

Article electronically published on October 20, 2009

\title{
MULTILINEAR OPERATORS WITH NON-SMOOTH KERNELS AND COMMUTATORS OF SINGULAR INTEGRALS
}

\author{
XUAN THINH DUONG, LOUKAS GRAFAKOS, AND LIXIN YAN
}

\begin{abstract}
We obtain endpoint estimates for multilinear singular integral operators whose kernels satisfy regularity conditions significantly weaker than those of the standard Calderón-Zygmund kernels. As a consequence, we deduce endpoint $L^{1} \times \cdots \times L^{1}$ to weak $L^{1 / m}$ estimates for the $m$ th-order commutator of Calderón. Our results reproduce known estimates for $m=1,2$ but are new for $m \geq 3$. We also explore connections between the 2nd-order higher-dimensional commutator and the bilinear Hilbert transform and deduce some new off-diagonal estimates for the former.
\end{abstract}

\section{INTRODUCTION}

Multilinear Calderón-Zygmund singular integral operators originated in the work of Coifman and Meyer [CM1], CM2], CM3]. The study of these operators has advanced significantly and although several features of its theory have presently been understood and developed, several other aspects of it remain unexplored.

In this work, we study boundedness properties of $m$-linear operators whose kernels have regularity significantly weaker than that of the standard CalderónZygmund kernels. For instance, our kernel regularity assumptions allow singularities along planes in $\mathbb{R}^{n}$. Consideration of these weak kernel conditions is motivated by the study of singular multilinear operators, such as the mth-order commutators, for which one would like to obtain an endpoint weak-type $L^{1 / m}$ estimate on $L^{1} \times \cdots \times L^{1}$. The results in this article have applications in the context of commutators of singular integrals; in fact the $m$ th-order commutator of Calderón falls under the scope of the theory developed herein. Endpoint estimates for these commutators on products of $L^{1}$ spaces have been obtained by C. Calderón [CC] when $m=1$ and Coifman and Meyer [CM1 when $m=1,2$, but the case $m \geq 3$ seems to have been missing from the literature and is completed in this work. We also study

Received by the editors January 28, 2008 and, in revised form, May 9, 2008.

2000 Mathematics Subject Classification. Primary 42B20, 42B25; Secondary 46B70, 47G30.

Key words and phrases. Multilinear operators, approximation to the identity, generalized Calderón-Zygmund kernel, Calderón-Zygmund decomposition, commutators.

The first author was supported by a grant from the Australia Research Council.

The second author was supported by grant DMS 0400387 of the US National Science Foundation and by the University of Missouri Research Council.

The third author was supported by NCET of Ministry of Education of China and NNSF of China (Grant No. 10771221). 
a connection between the higher-dimensional commutator introduced by Christ and Journé [CJ] and the bilinear Hilbert transforms and use this connection to obtain new off-diagonal bounds for the higher-dimensional commutator.

Before we formulate our results we set up some notation. We will follow the notation in GT1, GT2. These articles, and the references therein, contain some background on the subject.

Let $K\left(x, y_{1}, \cdots, y_{m}\right)$ be a locally integral function defined away from the diagonal $x=y_{1}=\cdots=y_{m}$ in $\left(\mathbb{R}^{n}\right)^{m+1}$, and let $T: \mathcal{S}\left(\mathbb{R}^{n}\right) \times \cdots \times \mathcal{S}\left(\mathbb{R}^{n}\right) \mapsto \mathcal{S}^{\prime}\left(\mathbb{R}^{n}\right)$ be an $m$-linear operator associated with the kernel $K\left(x, y_{1}, \cdots, y_{m}\right)$ in the following way:

$$
\begin{aligned}
& \left\langle T\left(f_{1}, \cdots, f_{m}\right), g\right\rangle \\
& \quad=\int_{\mathbb{R}^{n}} \int_{\left(\mathbb{R}^{n}\right)^{m}} K\left(x, y_{1}, \cdots, y_{m}\right) f_{1}\left(y_{1}\right) \cdots f_{m}\left(y_{m}\right) g(x) d y_{1} \cdots d y_{m} d x,
\end{aligned}
$$

where $f_{1}, \cdots, f_{m}, g$ in $\mathcal{S}\left(\mathbb{R}^{n}\right)$ with $\bigcap_{j=1}^{m} \operatorname{supp} f_{j} \cap \operatorname{supp} g=\emptyset$. We will assume the size estimate on the kernel $K$

$$
\left|K\left(x, y_{1}, \cdots, y_{j}, \cdots, y_{m}\right)\right| \leq \frac{A}{\left(\left|x-y_{1}\right|+\cdots+\left|x-y_{m}\right|\right)^{m n}}
$$

for some $A>0$ and all $\left(x, y_{1}, \cdots, y_{j}, \cdots, y_{m}\right)$ with $x \neq y_{j}$ for some $j$. We will also assume that for some $\epsilon>0$ we have the smoothness estimates

$$
\begin{gathered}
\left|K\left(x, y_{1}, \cdots, y_{j}, \cdots, y_{m}\right)-K\left(x^{\prime}, y_{1}, \cdots, y_{j}, \cdots, y_{m}\right)\right| \\
\leq \frac{A\left|x-x^{\prime}\right|^{\epsilon}}{\left(\left|x-y_{1}\right|+\cdots+\left|x-y_{m}\right|\right)^{m n+\epsilon}}
\end{gathered}
$$

whenever $\left|x-x^{\prime}\right| \leq \frac{1}{2} \max _{1 \leq j \leq m}\left|x-y_{j}\right|$ and also for each $j$,

$$
\begin{gathered}
\left|K\left(x, y_{1}, \cdots, y_{j}, \cdots, y_{m}\right)-K\left(x, y_{1}, \cdots, y_{j}^{\prime}, \cdots, y_{m}\right)\right| \\
\leq \frac{A\left|y_{j}-y_{j}^{\prime}\right|^{\epsilon}}{\left(\left|x-y_{1}\right|+\cdots+\left|x-y_{m}\right|\right)^{m n+\epsilon}}
\end{gathered}
$$

whenever $\left|y_{j}-y_{j}^{\prime}\right| \leq \frac{1}{2} \max _{1 \leq j \leq m}\left|x-y_{j}\right|$.

Under the above assumptions, we say that $T$ is an $m$-linear operator with Calderón-Zygmund kernel $K$. The collection of kernels $K$ satisfying (1.2), (1.3), and (1.4) with parameters $m, A$ and $\epsilon$ will be denoted by $m$ - $C Z K(A, \epsilon)$. Examples of operators with kernels in 2-CZK(A,1) are the bilinear Riesz transforms on $\mathbb{R} \times \mathbb{R}$, given for $j=1,2$ by

$$
R_{j}\left(f_{1}, f_{2}\right)(x)=\text { p.v. } \int_{\mathbb{R}} \int_{\mathbb{R}} \frac{x-y_{j}}{\left|\left(x-y_{1}, x-y_{2}\right)\right|^{3}} f_{1}\left(y_{1}\right) f_{2}\left(y_{2}\right) d y_{1} d y_{2} .
$$

Numerous other examples can be constructed. In fact, given a linear operator $P$ on $\mathbb{R}^{m n}$ with kernel in $1-C Z K(A, \epsilon)$, one may obtain an $m$-linear operator $T$ on $\mathbb{R}^{n} \times \cdots \times \mathbb{R}^{n}$ with kernel in $m-C Z K(A, \epsilon)$ by setting

$$
T\left(f_{1}, \cdots, f_{m}\right)(x)=P\left(f_{1} \otimes \cdots \otimes f_{m}\right)(x, \cdots, x)
$$


where $x \in \mathbb{R}^{n}$ and $(x, \cdots, x) \in \mathbb{R}^{m n}$ and $\left(f_{1} \otimes \cdots \otimes f_{m}\right)\left(x_{1}, \cdots, x_{m}\right)=f_{1}\left(x_{1}\right) \cdots$ $f_{m}\left(x_{m}\right)$.

For linear Calderón-Zygmund singular integrals one has the classical endpoint estimate $L^{1}\left(\mathbb{R}^{n}\right) \rightarrow L^{1, \infty}\left(\mathbb{R}^{n}\right)$. For $m$-linear operators, the corresponding endpoint result is as follows.

Theorem A. Let $T$ be a multilinear operator with a kernel $K$ in $m-C Z K(A, \epsilon)$. Assume that for some numbers $1 \leq q_{1}, q_{2}, \cdots, q_{m} \leq \infty$ and some $0<q<\infty$ satisfying

$$
\frac{1}{q_{1}}+\frac{1}{q_{2}}+\cdots+\frac{1}{q_{m}}=\frac{1}{q}
$$

$T$ maps $L^{q_{1}}\left(\mathbb{R}^{n}\right) \times \cdots \times L^{q_{m}}\left(\mathbb{R}^{n}\right)$ to $L^{q, \infty}\left(\mathbb{R}^{n}\right)$. Then $T$ can be extended to be a bounded operator from the $m$-fold product $L^{1}\left(\mathbb{R}^{n}\right) \times \cdots \times L^{1}\left(\mathbb{R}^{n}\right)$ to $L^{1 / m, \infty}\left(\mathbb{R}^{n}\right)$. Moreover, for some constant $C_{n, m}$ (that depends only on the parameters indicated) we have that

$$
\|T\|_{L^{1} \times \cdots \times L^{1} \rightarrow L^{1 / m, \infty}} \leq C_{n, m}\left(A+\|T\|_{L^{q_{1} \times \cdots \times L^{q_{m}} \rightarrow L^{q, \infty}}}\right) .
$$

It should be noted that $m$-linear operators $T$ that satisfy the hypotheses of Theorem A are usually called multilinear Calderón-Zygmund operators. For a proof of Theorem A, we refer to [GT2] and in some special cases, also to [KS.

We work with a class of integral operators $\left\{A_{t}\right\}_{t>0}$, which play the role of an approximation to the identity operator as in [DM]. We assume that the operators $A_{t}$ are associated with kernels $a_{t}(x, y)$ in the sense that

$$
A_{t} f(x)=\int_{\mathbb{R}^{n}} a_{t}(x, y) f(y) d y
$$

for every function $f \in L^{p}\left(\mathbb{R}^{n}\right), 1 \leq p \leq \infty$, and that the kernels $a_{t}(x, y)$ satisfy the following size conditions:

$$
\left|a_{t}(x, y)\right| \leq h_{t}(x, y)=t^{-n / s} h\left(\frac{|x-y|}{t^{1 / s}}\right),
$$

where $s$ is a positive fixed constant and $h$ is a positive, bounded, decreasing function satisfying

$$
\lim _{r \rightarrow \infty} r^{n+\eta} h\left(r^{s}\right)=0
$$

for some $\eta>0$. These conditions imply that for some $C^{\prime}>0$ and all $0<\eta^{\prime} \leq \eta$, the kernels $a_{t}(x, y)$ satisfy

$$
\left|a_{t}(x, y)\right| \leq C^{\prime} t^{-n / s}\left(1+t^{-1 / s}|x-y|\right)^{-n-\eta^{\prime}} .
$$

Now, let $T$ be a multilinear operator associated with a kernel $K\left(x, y_{1}, \cdots, y_{m}\right)$ in the sense of (1.1). The basic assumptions we are going to be making concerning $T$ are the following.

Assumption 2.1. Assume that for each $j=1,2, \cdots, m$, there exist operators $\left\{A_{t}^{(j)}\right\}_{t>0}$ with kernels $a_{t}^{(j)}(x, y)$ that satisfy conditions (1.5) and (1.6) with con- 
stants $s$ and $\eta$ and there exist kernels $K_{t}^{(j)}\left(x, y_{1}, \cdots, y_{m}\right)$ such that

$$
\begin{aligned}
& \left\langle T\left(f_{1}, \cdots, A_{t}^{(j)} f_{j}, \cdots, f_{m}\right), g\right\rangle \\
& \quad=\int_{\mathbb{R}^{n}} \int_{\left(\mathbb{R}^{n}\right)^{m}} K_{t}^{(j)}\left(x, y_{1}, \cdots, y_{m}\right) f_{1}\left(y_{1}\right) \cdots f_{m}\left(y_{m}\right) g(x) d y_{1} \cdots d y_{m} d x,
\end{aligned}
$$

for all $f_{1}, \cdots, f_{m}, g$ in $\mathcal{S}\left(\mathbb{R}^{n}\right)$ with $\bigcap_{j=1}^{m} \operatorname{supp} f_{j} \cap \operatorname{supp} g=\emptyset$.

Assumption 2.2. There exist a function $\phi \in C(\mathbb{R})$ with $\operatorname{supp} \phi \subset[-1,1]$ and a constant $\epsilon>0$ so that for all $x, y_{1}, \cdots, y_{m} \in \mathbb{R}^{n}$ and $t>0$ we have

$$
\begin{aligned}
\left|K\left(x, y_{1}, \cdots, y_{m}\right)-K_{t}^{(j)}\left(x, y_{1}, \cdots, y_{m}\right)\right| & \frac{A}{\left(\left|x-y_{1}\right|+\cdots+\left|x-y_{m}\right|\right)^{m n}} \sum_{\substack{k=1 \\
k \neq j}}^{m} \phi\left(\frac{\left|y_{j}-y_{k}\right|}{t^{1 / s}}\right) \\
& +\frac{A t^{\epsilon / s}}{\left(\left|x-y_{1}\right|+\cdots+\left|x-y_{m}\right|\right)^{m n+\epsilon}}
\end{aligned}
$$

for some $A>0$, whenever $t^{1 / s} \leq\left|x-y_{j}\right| / 2$.

Under Assumptions 2.1 and 2.2 we say that $T$ is an $m$-linear operator with generalized Calderón-Zygmund kernel $K$. The collection of functions $K$ satisfying (1.7) and (1.8) with parameters $m, A, s, \eta$ and $\epsilon$ will be denoted by $m-G C Z K_{0}(A, s, \eta, \epsilon)$.

The main goal of this article is to show that endpoint estimates can be obtained for operators associated with generalized Calderón-Zygmund kernels provided these operators are bounded on a single product of Lebesgue spaces. In particular, the most interesting endpoint case is the $m$-fold product of $L^{1}$ spaces. We have the following result:

Theorem 1.1. Let $T$ be a multilinear operator with a kernel $K$ in $m-G C Z K_{0}(A, s$, $\eta, \epsilon)$. Assume that for some $1 \leq q_{1}, q_{2}, \cdots, q_{m}<\infty$ and some $0<q<\infty$ with

$$
\frac{1}{q_{1}}+\frac{1}{q_{2}}+\cdots+\frac{1}{q_{m}}=\frac{1}{q}
$$

$T$ maps $L^{q_{1}}\left(\mathbb{R}^{n}\right) \times \cdots \times L^{q_{m}}\left(\mathbb{R}^{n}\right)$ to $L^{q, \infty}\left(\mathbb{R}^{n}\right)$. Then $T$ can be extended to be a bounded operator from the $m$-fold product $L^{1}\left(\mathbb{R}^{n}\right) \times \cdots \times L^{1}\left(\mathbb{R}^{n}\right)$ to $L^{1 / m, \infty}\left(\mathbb{R}^{n}\right)$.

Moreover, for some constant $C_{n, m}$ (that depends only on the parameters indicated) we have that

$$
\|T\|_{L^{1} \times \cdots \times L^{1} \rightarrow L^{1 / m, \infty}} \leq C_{n, m}\left(A+\|T\|_{L^{q_{1}} \times \cdots \times L^{q_{m}} \rightarrow L^{q, \infty}}\right) .
$$

As a consequence of Theorem 1.1 and multilinear interpolation, $T$ is also bounded from $L^{p_{1}}\left(\mathbb{R}^{n}\right) \times \cdots \times L^{p_{m}}\left(\mathbb{R}^{n}\right)$ to $L^{p}\left(\mathbb{R}^{n}\right)$ for all $1<p_{1}, \cdots, p_{m}<\infty$ and $1 / m<$ $p<\infty$ related to the $p_{j}$ as in (1.9). This will be discussed in Section 3 .

The layout of the paper is as follows. In Section 2, we give a proof of Theorem 1.1 and check that our condition is a consequence of the conditions of Theorem A (see Proposition 2.1 below). In Section 3, we discuss a multilinear interpolation theorem that allows us to remove the assumption $q_{j}<\infty$ on the indices in Theorem 1.1] In Section 4, we apply the results of Section 3 to deduce standard bounds for the $m$ th commutators of A. Calderón; these results seem to be new for $m \geq 3$. In Section 5, we obtain new bounds for the higher-dimensional commutator introduced by Christ and Journé by exploiting a connection between this commutator and the bilinear Hilbert transforms. 
Throughout, the letter " $C$ " will denote (possibly different) constants that are independent of the essential variables.

\section{Proof of Theorem 1.1}

For matters of simplicity, we assume that for all $j$ the operators $A_{t}^{(j)}$ all coincide with a fixed operator $A_{t}$ with kernel $a_{t}(x, y)$ which satisfies conditions (1.5) and (1.6) with two positive constants $s$ and $\eta>\epsilon$, where $\epsilon$ is the constant in (1.8).

Proof of Theorem 1.1. Setting $B=\|T\|_{L^{q_{1}} \times \ldots \times L^{q_{m}} \rightarrow L^{q, \infty}}$, for $1 \leq i \leq m$, we fix $f_{i} \in L^{1}\left(\mathbb{R}^{n}\right)$, and by the linearity of the operator, we may assume that $\left\|f_{i}\right\|_{L^{1}\left(\mathbb{R}^{n}\right)}=$ 1. Also fix a $\lambda>0$. We would like to show that there exists a constant $C>0$ such that

$$
\left|\left\{x \in \mathbb{R}^{n}:\left|T\left(f_{1}, \ldots, f_{m}\right)(x)\right|>\lambda\right\}\right| \leq C(A+B)^{1 / m} \lambda^{-1 / m} .
$$

Once (2.1) has been established for $f_{i}$ 's with norm one, the general case follows immediately by scaling. Let $\alpha$ be a positive real number to be determined later. We perform a Calderón-Zygmund decomposition of functions $\left\{f_{i}\right\}_{i=1}^{m}$ at height $(\alpha \lambda)^{1 / m}$ to obtain a decomposition

$$
f_{i}=g_{i}+b_{i}=g_{i}+\sum_{k} b_{i, k}
$$

such that

(a) $\left|g_{i}(x)\right| \leq C(\alpha \lambda)^{1 / m}$ for all almost $x \in \mathbb{R}^{n}$;

(b) there exists a sequence of pairwise disjoint cubes $\left\{Q_{i, k}\right\}_{k}$ so that the support of each $b_{i, k}$ is contained in $Q_{i, k}$, and

$$
\int_{\mathbb{R}^{n}}\left|b_{i, k}(x)\right| d x \leq C(\alpha \lambda)^{1 / m}\left|Q_{i, k}\right|
$$

(c) $\sum_{k}\left|Q_{i, k}\right| \leq C(\alpha \lambda)^{-1 / m}$.

Note that the functions $b_{i, k}$ are usually chosen to satisfy $\int_{\mathbb{R}^{n}} b_{i, k}(x) d x=0$ as well, but we do not need this property. Conditions (b) and (c) imply that $\left\|b_{i}\right\|_{L^{1}\left(\mathbb{R}^{n}\right)} \leq C$ and hence that $\left\|g_{i}\right\|_{L^{1}\left(\mathbb{R}^{n}\right)} \leq 1+C$. In addition, from condition (a) we have that $\left\|g_{i}\right\|_{L^{p}\left(\mathbb{R}^{n}\right)} \leq C(\alpha \lambda)^{1 / m p^{\prime}}$ for $1 \leq p \leq \infty\left(p^{\prime}\right.$ is here the dual exponent of $\left.p\right)$. Now let

$$
\begin{aligned}
E_{\lambda}^{(1)} & =\left\{x \in \mathbb{R}^{n}:\left|T\left(g_{1}, g_{2}, \ldots, g_{m}\right)(x)\right|>\lambda / 2^{m}\right\}, \\
E_{\lambda}^{(2)} & =\left\{x \in \mathbb{R}^{n}:\left|T\left(b_{1}, g_{2}, \ldots, g_{m}\right)(x)\right|>\lambda / 2^{m}\right\}, \\
E_{\lambda}^{(3)} & =\left\{x \in \mathbb{R}^{n}:\left|T\left(g_{1}, b_{2}, \ldots, g_{m}\right)(x)\right|>\lambda / 2^{m}\right\}, \\
\ldots & \ldots \\
E_{\lambda}^{\left(2^{m}\right)} & =\left\{x \in \mathbb{R}^{n}:\left|T\left(b_{1}, b_{2}, \ldots, b_{m}\right)(x)\right|>\lambda / 2^{m}\right\},
\end{aligned}
$$

where each $E_{\lambda}^{(s)}=\left|\left\{x \in \mathbb{R}^{n}:\left|T\left(h_{1}, h_{2}, \ldots, h_{m}\right)(x)\right|>\lambda / 2^{m}\right\}\right|$ with $h_{j} \in\left\{g_{j}, b_{j}\right\}$ and all the sets $E_{\lambda}^{(s)}$ are distinct. Since $\left|\left\{x \in \mathbb{R}^{n}:\left|T\left(f_{1}, f_{2}, \ldots, f_{m}\right)(x)\right|>\lambda\right\}\right| \leq$ $\sum_{i=1}^{2^{m}}\left|E_{\lambda}^{(i)}\right|$, it will suffice to prove estimate (2.1) for each of the $2^{m}$ sets $E_{\lambda}^{(s)}$. 
For the sake of clarity, we first present the proof when $m=2$. Let us start estimating the measure of the set $E_{\lambda}^{(1)}$, which is the easiest. Chebychev's inequality and the $L^{q_{1}}\left(\mathbb{R}^{n}\right) \times L^{q_{2}}\left(\mathbb{R}^{n}\right) \rightarrow L^{q, \infty}\left(\mathbb{R}^{n}\right)$ boundedness of $T$ give

$$
\begin{aligned}
\left|E_{\lambda}^{(1)}\right| & \leq(4 B)^{q} \lambda^{-q}\left\|g_{1}\right\|_{L^{q_{1}\left(\mathbb{R}^{n}\right)}}^{q}\left\|g_{2}\right\|_{L^{q_{2}\left(\mathbb{R}^{n}\right)}}^{q} \\
& \leq C B^{q} \lambda^{-q}(\alpha \lambda)^{\left(2-\frac{1}{q}\right) \frac{q}{2}} \\
& \leq C^{\prime} B^{q} \lambda^{-1 / 2} \alpha^{q-\frac{1}{2}} .
\end{aligned}
$$

Consider now the term $E_{\lambda}^{(s)}$ as above with $s=2,3,4$. We will show that

$$
\left|E_{\lambda}^{(s)}\right| \leq C B^{q} \lambda^{-1 / 2} \alpha^{q-\frac{1}{2}}+C \lambda^{-1 / 2}\left(\alpha^{-1 / 2}+A \alpha^{1 / 2}\right) .
$$

Let $x_{Q_{i, k}}$ be the center of the cube $Q_{i, k}$ and let $\ell\left(Q_{i, k}\right)$ be the side length of $Q_{i, k}$. Fix an $\epsilon>0$ in (1.8). We denote by

$$
\mathcal{J}_{i, \epsilon}(x)=\sum_{k} \frac{\left(\ell\left(Q_{i, k}\right)\right)^{n+\epsilon}}{\left(\ell\left(Q_{i, k}\right)+\left|x-x_{Q_{i, k}}\right|\right)^{n+\epsilon}}, \quad i=1,2,
$$

the Marcinkiewicz function associated with the family of the cubes $\left\{Q_{i, k}\right\}_{k}$. It is a known fact, see [FS], that for every $p>n /(n+\epsilon)$ we have

$$
\left\|\mathcal{J}_{i, \epsilon}\right\|_{L^{p}\left(\mathbb{R}^{n}\right)} \leq C\left(\sum_{k}\left|Q_{i, k}\right|\right)^{1 / p} \leq C(\alpha \lambda)^{-\frac{1}{2 p}} .
$$

For the term $E_{\lambda}^{(2)}$, we note that $b_{1}=\sum_{k} b_{1, k}$ in which every $b_{1, k}$ is supported in a cube $Q_{1, k}$. We will approximate each $b_{1, k}$ by $A_{t_{1, k}} b_{1, k}$, where $t_{1, k}=\left(\ell\left(Q_{1, k}\right)\right)^{s}$ and $s$ is the constant appearing in (1.5), and decompose

$$
\begin{aligned}
T\left(b_{1}, g_{2}\right)(x) & =T\left(\sum_{k} b_{1, k}, g_{2}\right)(x) \\
& =T\left(\sum_{k} A_{t_{1, k}} b_{1, k}, g_{2}\right)(x)+T\left(\sum_{k}\left(b_{1, k}-A_{t_{1, k}} b_{1, k}\right), g_{2}\right)(x) .
\end{aligned}
$$

For the first of the above terms, we note that if $x \in \mathbb{R}^{n}$ and $y \in Q_{1, k}$, then

$$
\ell\left(Q_{1, k}\right)+|x-y| \approx \ell\left(Q_{1, k}\right)+\left|x-x_{Q_{1, k}}\right| .
$$

Using conditions (1.5), (1.6) and property (b) of the Calderón-Zygmund decomposition, we have

$$
\begin{aligned}
\left|\sum_{k} A_{t_{1, k}} b_{1, k}(x)\right| & \leq C \sum_{k} \int_{\mathbb{R}^{n}} \frac{\left(\ell\left(Q_{1, k}\right)\right)^{\epsilon}}{\left(\ell\left(Q_{1, k}\right)+|x-y|\right)^{n+\epsilon}}\left|b_{1, k}(y)\right| d y \\
& \leq C \sum_{k} \frac{\left(\ell\left(Q_{1, k}\right)\right)^{\epsilon}}{\left(\ell\left(Q_{1, k}\right)+\left|x-x_{Q_{1, k}}\right|\right)^{n+\epsilon}}\left\|b_{1, k}\right\|_{L^{1}\left(\mathbb{R}^{n}\right)} \\
& \leq C(\alpha \lambda)^{1 / 2} \mathcal{J}_{1, \epsilon}(x),
\end{aligned}
$$


where we have used the fact that $0<\epsilon<\eta$ and the size estimate for the decreasing function $h$. Using (2.6) we obtain

$$
\begin{aligned}
\mid\{x & \left.\in \mathbb{R}^{n}:\left|T\left(\sum_{k} A_{t_{1, k}} b_{1, k}, g_{2}\right)(x)\right|>\frac{\lambda}{4}\right\} \mid \\
& \leq(4 B)^{q} \lambda^{-q}\left\|\sum_{k} A_{t_{1, k}} b_{1, k}\right\|_{L^{q_{1}\left(\mathbb{R}^{n}\right)}}^{q}\left\|g_{2}\right\|_{L^{q_{2}\left(\mathbb{R}^{n}\right)}}^{q} \\
& \leq C \lambda^{-q} B^{q}(\alpha \lambda)^{q / 2}\left\|\mathcal{J}_{1, \epsilon}\right\|_{L^{q_{1}\left(\mathbb{R}^{n}\right)}}^{q}(\alpha \lambda)^{\frac{q}{2}\left(1-\frac{1}{q_{2}}\right)} \\
& \leq C \lambda^{-q} B^{q}(\alpha \lambda)^{\frac{q}{2}\left(1-\frac{1}{q_{1}}\right)}(\alpha \lambda)^{\frac{q}{2}\left(1-\frac{1}{q_{2}}\right)} \\
& \leq C^{\prime} B^{q} \lambda^{-1 / 2} \alpha^{q-\frac{1}{2}} .
\end{aligned}
$$

Now, we let

$$
\Omega_{i}^{*}=\bigcup_{k} Q_{i, k}^{*}, \quad i=1,2,
$$

where $Q_{i, k}^{*}$ is a cube with the same center as $Q_{i, k}$ but expanded 5 times. By property (c) of the Calderón-Zygmund decomposition, we have

$$
\left|\Omega_{i}^{*}\right|=\left|\bigcup_{k} Q_{i, k}^{*}\right| \leq C \sum_{k}\left|Q_{i, k}\right| \leq C(\alpha \lambda)^{-1 / 2}
$$

Then,

$$
\begin{aligned}
& \left|\left\{x \in \mathbb{R}^{n}:\left|\sum_{k} T\left(b_{1, k}-A_{t_{1, k}} b_{1, k}, g_{2}\right)(x)\right|>\frac{\lambda}{4}\right\}\right| \\
& \leq C(\alpha \lambda)^{-1 / 2}+\frac{4}{\lambda} \sum_{k} \int_{\left(Q_{1, k}^{*}\right)^{c}}\left|T\left(b_{1, k}-A_{t_{1, k}} b_{1, k}, g_{2}\right)(x)\right| d x .
\end{aligned}
$$

Next we observe that for $x \notin Q_{1, k}^{*}$ and $y_{1} \in Q_{1, k}$, we have that

$$
\frac{1}{2}\left|x-y_{1}\right|>\ell\left(Q_{1, k}\right)=t_{1, k}^{1 / s}
$$

and that

$$
\left|x-y_{1}\right|+\left|x-y_{2}\right| \geq(2+\sqrt{n} / 2)^{-1}\left(\left|x-y_{1}\right|+\sqrt{n} \ell\left(Q_{1, k}\right)+\left|y_{1}-y_{2}\right|\right) .
$$

These facts, together with condition (1.8), yield

$$
\begin{aligned}
& \sum_{k} \int_{\left(Q_{1, k}^{*}\right)^{c}}\left|T\left(b_{1, k}-A_{t_{1, k}} b_{1, k}, g_{2}\right)(x)\right| d x \\
& \leq \sum_{k} \int_{\left(\mathbb{R}^{n}\right)^{2}}\left(\int_{\left(Q_{1, k}^{*}\right)^{c}}\left|K\left(x, y_{1}, y_{2}\right)-K_{t_{1, k}}^{(1)}\left(x, y_{1}, y_{2}\right)\right| d x\right)\left|b_{1, k}\left(y_{1}\right)\right|\left|g_{2}\left(y_{2}\right)\right| d y_{1} d y_{2} \\
& \leq C A \sum_{k} \int_{\left(\mathbb{R}^{n}\right)^{2}}\left(\int_{\mathbb{R}^{n}} \frac{\ell\left(Q_{1, k}\right)^{\epsilon}}{\left(\left|x-y_{1}\right|+\sqrt{n} \ell\left(Q_{1, k}\right)+\left|y_{1}-y_{2}\right|\right)^{2 n+\epsilon}} d x\right)\left|b_{1, k}\left(y_{1}\right)\right|\left|g_{2}\left(y_{2}\right)\right| d y_{1} d y_{2} \\
& +C A \sum_{k} \int_{\left(\mathbb{R}^{n}\right)^{3}} \frac{1}{\left(\left|x-y_{2}\right|+\sqrt{n} \ell\left(Q_{1, k}\right)\right)^{2 n}} \phi\left(\frac{\left|y_{1}-y_{2}\right|}{\ell\left(Q_{1, k}\right)}\right)\left|b_{1, k}\left(y_{1}\right)\right|\left|g_{2}\left(y_{2}\right)\right| d y_{1} d y_{2} d x \\
& =I+I I .
\end{aligned}
$$


For term $I$, we have

$$
\begin{aligned}
I & \leq C A \sum_{k} \int_{\left(\mathbb{R}^{n}\right)^{2}} \frac{\ell\left(Q_{1, k}\right)^{\epsilon}}{\left(\sqrt{n} \ell\left(Q_{1, k}\right)+\left|y_{1}-y_{2}\right|\right)^{n+\epsilon}}\left|b_{1, k}\left(y_{1}\right)\right|\left|g_{2}\left(y_{2}\right)\right| d y_{1} d y_{2} \\
& \leq C A \sum_{k} \int_{\left.\mathbb{R}^{n}\right)^{2}} \frac{\ell\left(Q_{1, k}\right)^{\epsilon}}{\left(\frac{1}{2} \sqrt{n} \ell\left(Q_{1, k}\right)+\left|x_{Q_{1, k}}-y_{2}\right|\right)^{n+\epsilon}}\left|b_{1, k}\left(y_{1}\right)\right|\left|g_{2}\left(y_{2}\right)\right| d y_{1} d y_{2} \\
& \leq C A(\alpha \lambda)^{1 / 2} \int_{\mathbb{R}^{n}}\left|g_{2}\left(y_{2}\right)\right| \mathcal{J}_{1, \epsilon}\left(y_{2}\right) d y_{2} \\
& \leq C A(\alpha \lambda)^{1 / 2}\left\|g_{2}\right\|_{L^{2}\left(\mathbb{R}^{n}\right)}\left\|\mathcal{J}_{1, \epsilon}\right\|_{L^{2}\left(\mathbb{R}^{n}\right)} \leq C A(\alpha \lambda)^{1 / 2} .
\end{aligned}
$$

Consider term II. We use the hypothesis that $\phi$ is supported in $[-1,1]$ to obtain

$$
\begin{aligned}
I I & \leq C A \sum_{k}\left\|b_{1, k}\right\|_{L^{1}\left(\mathbb{R}^{n}\right)} \int_{\left(\mathbb{R}^{n}\right)^{2}} \frac{1}{\left(\ell\left(Q_{1, k}\right)+\left|x-y_{2}\right|\right)^{2 n}}\left|g_{2}\left(y_{2}\right)\right| \mathbf{1}_{\left(Q_{1, k}\right)^{*}}\left(y_{2}\right) d y_{2} d x \\
& \leq C A(\alpha \lambda)^{1 / 2} \sum_{k} \int_{\left(\mathbb{R}^{n}\right)^{2}} \frac{\ell\left(Q_{1, k}\right)^{n}}{\left(\ell\left(Q_{1, k}\right)+\left|x-y_{2}\right|\right)^{2 n}}\left|g_{2}\left(y_{2}\right)\right| \mathbf{1}_{\left(Q_{1, k}\right)^{*}}\left(y_{2}\right) d y_{2} d x \\
& \leq C A(\alpha \lambda)^{1 / 2} \int_{\mathbb{R}^{n}}\left|g_{2}\left(y_{2}\right)\right| \mathbf{1}_{\cup_{k}\left(Q_{1, k}\right)^{*}}\left(y_{2}\right) d y_{2} \\
& \leq C A(\alpha \lambda)^{1 / 2}\left\|g_{2}\right\|_{L^{2}\left(\mathbb{R}^{n}\right)}\left|\bigcup_{k}\left(Q_{1, k}\right)^{*}\right|^{1 / 2} \leq C A(\alpha \lambda)^{1 / 2} .
\end{aligned}
$$

Estimates for $I$ and $I I$, combined with (2.9) and (2.8), yield

$$
\left|E_{\lambda}^{(2)}\right| \leq C \lambda^{-1 / 2}\left(\alpha^{-1 / 2}+A \alpha^{1 / 2}\right) .
$$

By symmetry, we obtain that $\left|E_{\lambda}^{(3)}\right| \leq C \lambda^{-1 / 2}\left(\alpha^{-1 / 2}+A \alpha^{1 / 2}\right)$.

Finally, we estimate term $E_{\lambda}^{(4)}$. For this one, we use an approach as we employed for terms $E_{\lambda}^{(2)}$ to decompose

$$
T\left(b_{1}, b_{2}\right)(x)=T\left(\sum_{k} b_{1, k}, \sum_{j} b_{2, j}\right)(x)=: \sum_{i=1}^{5} T_{i}\left(b_{1}, b_{2}\right)(x),
$$

where

$$
\begin{aligned}
T_{1}\left(b_{1}, b_{2}\right)(x) & =T\left(\sum_{k} A_{t_{1, k}} b_{1, k}, \sum_{j} A_{t_{2, j}} b_{2, j}\right)(x), \\
T_{2}\left(b_{1}, b_{2}\right)(x) & =\sum_{k} \sum_{j: \ell\left(Q_{1, k}\right) \leq \ell\left(Q_{2, j}\right)} T\left(A_{t_{1, k}} b_{1, k}, b_{2, j}-A_{t_{2, j}} b_{2, j}\right)(x), \\
T_{3}\left(b_{1}, b_{2}\right)(x) & =\sum_{k} \sum_{j: \ell\left(Q_{1, k}\right) \leq \ell\left(Q_{2, j}\right)} T\left(b_{1, k}-A_{t_{1, k}} b_{1, k}, b_{2, j}\right)(x), \\
T_{4}\left(b_{1}, b_{2}\right)(x) & =\sum_{k} \sum_{j: \ell\left(Q_{1, k}\right)>\ell\left(Q_{2, j}\right)} T\left(b_{1, k}-A_{t_{1, k}} b_{1, k}, A_{t_{2, j}} b_{2, j}\right)(x), \\
T_{5}\left(b_{1}, b_{2}\right)(x) & =\sum_{k} \sum_{j: \ell\left(Q_{1, k}\right)>\ell\left(Q_{2, j}\right)} T\left(b_{1, k}, b_{2, j}-A_{t_{2, j}} b_{2, j}\right)(x) .
\end{aligned}
$$

For terms $T_{1}\left(b_{1}, b_{2}\right)$ and $T_{2}\left(b_{1}, b_{2}\right)$ we can use an argument similar to that in the treatment of $E_{\lambda}^{(2)}$ to show that

$$
\left|\left\{x \in \mathbb{R}^{n}:\left|T_{1}\left(b_{1}, b_{2}\right)(x)\right|>\lambda / 20\right\}\right| \leq C B^{q} \lambda^{-1 / 2} \alpha^{q-\frac{1}{2}}
$$


and

$$
\left|\left\{x \in \mathbb{R}^{n}:\left|T_{2}\left(b_{1}, b_{2}\right)(x)\right|>\lambda / 20\right\}\right| \leq C \lambda^{-1 / 2}\left(\alpha^{-1 / 2}+A \alpha^{1 / 2}\right) .
$$

Now consider term $T_{3}\left(b_{1}, b_{2}\right)(x)$. By condition (1.8), we have

$$
\begin{aligned}
& \left|T_{3}\left(b_{1}, b_{2}\right)(x)\right| \\
& \leq \sum_{k} \sum_{j: \ell\left(Q_{1, k}\right) \leq \ell\left(Q_{2, j}\right)} \int_{\left(\mathbb{R}^{n}\right)^{2}}\left|\left[K\left(x, y_{1}, y_{2}\right)-K_{t_{1, k}}^{(1)}\left(x, y_{1}, y_{2}\right)\right] b_{1, k}\left(y_{1}\right) b_{2, j}\left(y_{2}\right)\right| d y_{1} d y_{2} \\
& \leq C A \sum_{k} \sum_{j: \ell\left(Q_{1, k}\right) \leq \ell\left(Q_{2, j}\right)} \int_{\left(\mathbb{R}^{n}\right)^{2}} \frac{\ell\left(Q_{1, k}\right)^{\epsilon}}{\left(\left|x-y_{1}\right|+\left|x-y_{2}\right|\right)^{2 n+\epsilon}}\left|b_{1, k}\left(y_{1}\right) b_{2, j}\left(y_{2}\right)\right| d y_{1} d y_{2} \\
& +C A \sum_{k} \sum_{j: \ell\left(Q_{1, k}\right) \leq \ell\left(Q_{2, j}\right)} \int_{\left(\mathbb{R}^{n}\right)^{2}} \frac{\left|b_{1, k}\left(y_{1}\right) b_{2, j}\left(y_{2}\right)\right|}{\left(\left|x-y_{1}\right|+\left|x-y_{2}\right|\right)^{2 n}} \phi\left(\frac{\left|y_{1}-y_{2}\right|}{\ell\left(Q_{1, k}\right)}\right) d y_{1} d y_{2} \\
& =: T_{31}\left(b_{1}, b_{2}\right)(x)+T_{32}\left(b_{1}, b_{2}\right)(x) .
\end{aligned}
$$

Note that if $x \notin \bigcup_{i=1}^{2} \Omega_{i}^{*}=\bigcup_{i=1}^{2} \bigcup_{k}\left(Q_{i, k}\right)^{*}$ and $y_{i} \in Q_{i, k}$, then

$$
\left|x-y_{i}\right| \approx \ell\left(Q_{i, k}\right)+\left|x-x_{Q_{i, k}}\right| \text {. }
$$

The assumption $\ell\left(Q_{1, k}\right) \leq \ell\left(Q_{2, j}\right)$ implies

$$
\begin{aligned}
& T_{31}\left(b_{1}, b_{2}\right)(x) \\
& \leq C A \int_{\left(\mathbb{R}^{n}\right)^{2}}\left(\sum_{k} \frac{\ell\left(Q_{1, k}\right)^{\frac{\epsilon}{2}}\left|b_{1, k}\left(y_{1}\right)\right|}{\left(\ell\left(Q_{1, k}\right)+\left|x-y_{1}\right|\right)^{n+\frac{\epsilon}{2}}}\right)\left(\sum_{j} \frac{\ell\left(Q_{2, j}\right)^{\frac{\epsilon}{2}}\left|b_{2, j}\left(y_{2}\right)\right|}{\left(\ell\left(Q_{2, j}\right)+\left|x-y_{2}\right|\right)^{n+\frac{\epsilon}{2}}}\right) d y_{1} d y_{2} \\
& \leq C A(\alpha \lambda) \mathcal{J}_{1, \epsilon / 2}(x) \mathcal{J}_{2, \epsilon / 2}(x),
\end{aligned}
$$

which gives

$$
\begin{aligned}
\int_{\left(\cup_{i=1}^{2} \Omega_{i}^{*}\right)^{c}}\left|T_{31}\left(b_{1}, b_{2}\right)(x)\right| d x & \leq C A(\alpha \lambda) \int_{\mathbb{R}^{n}} \mathcal{J}_{1, \epsilon / 2}(x) \mathcal{J}_{2, \epsilon / 2}(x) d x \\
& \leq C A(\alpha \lambda)\left\|\mathcal{J}_{1, \epsilon / 2}\right\|_{L^{2}\left(\mathbb{R}^{n}\right)}\left\|\mathcal{J}_{2, \epsilon / 2}\right\|_{L^{2}\left(\mathbb{R}^{n}\right)} \\
& \leq C A(\alpha \lambda)^{1 / 2} .
\end{aligned}
$$

On the other hand, since $\left\{Q_{1, k}\right\}_{k}$ is a sequence of pairwise disjoint cubes in $\mathbb{R}^{n}$, we use the fact that $\phi$ is supported in $[-1,1]$, and the assumption $\ell\left(Q_{1, k}\right) \leq \ell\left(Q_{2, j}\right)$, to deduce

$$
\begin{aligned}
& \left|T_{32}\left(b_{1}, b_{2}\right)(x)\right| \\
& \leq C A \sum_{k} \sum_{j: \ell\left(Q_{1, k}\right) \leq \ell\left(Q_{2, j}\right)}\left\|b_{1, k}\right\|_{L^{1}\left(\mathbb{R}^{n}\right)} \int_{\mathbb{R}^{n}} \frac{1}{\left(\ell\left(Q_{2, j}\right)+\left|x-y_{2}\right|\right)^{2 n}}\left|b_{2, j}\left(y_{2}\right)\right| \mathbf{1}_{\left(Q_{1, k}\right)^{*}}\left(y_{2}\right) d y_{2} \\
& \leq C A(\alpha \lambda)^{1 / 2} \sum_{j} \int_{\mathbb{R}^{n}} \frac{\ell\left(Q_{2, j}\right)^{n}}{\left(\ell\left(Q_{2, j}\right)+\left|x-y_{2}\right|\right)^{2 n}}\left|b_{2, j}\left(y_{2}\right)\right| \mathbf{1}_{\cup_{k}\left(Q_{1, k}\right)^{*}}\left(y_{2}\right) d y_{2} \\
& \leq C A(\alpha \lambda)^{1 / 2} \sum_{j} \int_{\mathbb{R}^{n}} \frac{\ell\left(Q_{2, j}\right)^{n}}{\left(\ell\left(Q_{2, j}\right)+\left|x-y_{2}\right|\right)^{2 n}}\left|b_{2, j}\left(y_{2}\right)\right| d y_{2} .
\end{aligned}
$$


This gives

$$
\begin{aligned}
& \int_{\left(\cup_{i=1}^{2} \Omega_{i}^{*}\right)^{c}}\left|T_{32}\left(b_{1}, b_{2}\right)(x)\right| d x \\
& \quad \leq C A(\alpha \lambda)^{1 / 2} \sum_{j} \int_{\left(\mathbb{R}^{n}\right)^{2}} \frac{\ell\left(Q_{2, j}\right)^{n}}{\left(\ell\left(Q_{2, j}\right)+\left|x-y_{2}\right|\right)^{2 n}}\left|b_{2, j}\left(y_{2}\right)\right| d y_{2} d x \\
& \quad \leq C A(\alpha \lambda)^{1 / 2} \sum_{j} \int_{\mathbb{R}^{n}}\left|b_{2, j}\left(y_{2}\right)\right| d y_{2} \\
& \quad \leq C A(\alpha \lambda)^{1 / 2} .
\end{aligned}
$$

Therefore,

$$
\begin{aligned}
\left|\left\{x \in \mathbb{R}^{n}:\left|T_{3}\left(b_{1}, b_{2}\right)(x)\right|>\frac{\lambda}{20}\right\}\right| & \leq\left|\bigcup_{i=1}^{2} \Omega_{i}^{*}\right|+\frac{20}{\lambda} \sum_{i=1}^{2} \int_{\left(\cup_{i=1}^{2} \Omega_{i}^{*}\right)^{c}}\left|T_{3 i}\left(b_{1}, b_{2}\right)(x)\right| d x \\
& \leq C \lambda^{-1 / 2}\left(\alpha^{-1 / 2}+A \alpha^{1 / 2}\right) .
\end{aligned}
$$

By symmetry, we have the same estimates for the terms $T_{4}\left(b_{1}, b_{2}\right)$ and $T_{5}\left(b_{1}, b_{2}\right)$, and then the desired estimate $E_{\lambda}^{(4)}$ follows readily. Combining estimates of $\left\{E_{\lambda}^{(i)}\right\}_{i=1}^{4}$, we obtain estimate (2.4) for any $\alpha>0$. Selecting $\alpha=(A+B)^{-1}$ in both (2.3) and (2.4) we obtain the estimate (2.1) when $m=2$.

Consider the case $m \geq 3$. Chebychev's inequality and the $L^{q_{1}}\left(\mathbb{R}^{n}\right) \times \ldots \times$ $L^{q_{m}}\left(\mathbb{R}^{n}\right) \rightarrow L^{q, \infty}\left(\mathbb{R}^{n}\right)$ boundedness of $T$ give $E_{\lambda}^{(1)} \leq C B^{q} \lambda^{-\frac{1}{m}} \alpha^{q-\frac{1}{m}}$. Let us estimate a set $E_{\lambda}^{(s)}$ of (2.2) with $2 \leq s \leq 2^{m}$. Suppose that for some $1 \leq l \leq m$ we have $l$ bad functions and $m-l$ good functions appearing in $T\left(h_{1}, \ldots, h_{m}\right)$, where $h_{j} \in\left\{g_{j}, b_{j}\right\}$. It suffices to show that

$$
\left|E_{\lambda}^{(s)}\right| \leq C B^{q} \lambda^{-\frac{1}{m}} \alpha^{q-\frac{1}{m}}+C \lambda^{-\frac{1}{m}}\left(\alpha^{-\frac{1}{m}}+A \alpha^{1-\frac{1}{m}}\right) .
$$

For matters of simplicity, we assume that the bad functions appear at the entries $1,2, \ldots, l$, i.e., $E_{\lambda}^{\left(s_{l}\right)}=T\left(b_{1}, \ldots, b_{l}, g_{l+1}, \ldots, g_{m}\right)$, where $s_{l}=\sum_{i=0}^{l-1} C_{m}^{i}+1$. Fix an $x \notin \bigcup_{j=1}^{l} \bigcup_{k_{j}}\left(Q_{j, k_{j}}\right)^{*}$. One has

$$
\begin{aligned}
T\left(b_{1}, \ldots, b_{l}, g_{l+1}, \ldots, g_{m}\right)(x) & \\
= & \sum_{k_{1}, \ldots, k_{l}} T\left(b_{1, k_{1}}, \ldots, b_{l, k_{l}}, g_{l+1}, \ldots, g_{m}\right)(x) \\
= & \sum_{k_{2}, \ldots, k_{l}} \sum_{\substack{k_{1}: \ell\left(Q_{1, k_{1}}\right) \leq \ell\left(Q_{i, k_{i}}\right) \\
\text { for all } i=2, \ldots, l}} T\left(b_{1, k_{1}}, \ldots, b_{l, k_{l}}, g_{l+1}, \ldots, g_{m}\right)(x) \\
& +\sum_{j=2}^{l} \sum_{\substack{k_{1}, \ldots, k_{j-1} \\
k_{j+1}, \ldots, k_{l}}} \sum_{k_{j}: k_{j} \in \Theta_{j}} T\left(b_{1, k_{1}}, \ldots, b_{l, k_{l}}, g_{l+1}, \ldots, g_{m}\right)(x) \\
= & : \sum_{j=1}^{l} T^{(j)}\left(b_{1}, \ldots, b_{l}, g_{l+1}, \ldots, g_{m}\right)(x),
\end{aligned}
$$

where $\Theta_{j}$ denotes the set

$$
\left\{k_{j}: \ell\left(Q_{j, k_{j}}\right)<\ell\left(Q_{i, k_{i}}\right), i=1, \ldots, j-1 \text { and } \ell\left(Q_{j, k_{j}}\right) \leq \ell\left(Q_{i, k_{i}}\right), i=j+1, \ldots, l\right\} .
$$


For every $T^{(j)}\left(b_{1}, \ldots, b_{l}, g_{l+1}, \ldots, g_{m}\right)$, we will approximate each $b_{j, k_{j}}$ by $A_{t_{j, k_{j}}} b_{j, k_{j}}$, where $t_{j, k_{j}}=\left(\ell\left(Q_{j, k_{j}}\right)\right)^{s}$ and $s$ is the constant appearing in (1.5); e.g.,

$$
\begin{aligned}
T^{(1)}\left(b_{1}, \ldots, b_{l}, g_{l+1}, \ldots, g_{m}\right)(x) & \sum_{k_{2}, \ldots, k_{l}} \sum_{\substack{k_{1}: \ell\left(Q_{1, k_{1}}\right) \leq \ell\left(Q_{i, k_{i}}\right) \\
\text { for all } i=2, \ldots, l}} T\left(b_{1, k_{1}}-A_{t_{1, k_{1}}}, b_{1, k_{1}}, \ldots, b_{l, k_{l}}, g_{l+1}, \ldots, g_{m}\right)(x) \\
& +\sum_{\substack{k_{2}, \ldots, k_{l} \\
k_{1}: \ell\left(Q_{1, k_{1}}\right) \leq \ell\left(Q_{i, k_{i}}\right) \\
\text { for all }}} T\left(A_{t_{1, k_{1}}} b_{1, k_{1}, \ldots, l}, \ldots, b_{l, k_{l}}, g_{l+1}, \ldots, g_{m}\right)(x) \\
(2.14) & =: \quad T^{(11)}\left(b_{1}, \ldots, b_{l}, g_{l+1}, \ldots, g_{m}\right)(x)+T^{(12)}\left(b_{1}, \ldots, b_{l}, g_{l+1}, \ldots, g_{m}\right)(x) .
\end{aligned}
$$

Consider the term $T^{(11)}\left(b_{1}, \ldots, b_{l}, g_{l+1}, \ldots, g_{m}\right)$. Note that $x \notin \bigcup_{j=1}^{l} \bigcup_{k_{j}}\left(Q_{j, k_{j}}\right)^{*}$. By condition (1.8), we have

$$
\begin{aligned}
& \left|T^{(11)}\left(b_{1}, \ldots, b_{l}, g_{l+1}, \ldots, g_{m}\right)(x)\right| \\
& \leq C A \sum_{k_{2}, \ldots, k_{l}} \sum_{\substack{k_{1}: \ell\left(Q_{1, k_{1}}\right) \leq \ell\left(Q_{i, k_{i}}\right) \\
\text { for all } i=2, \ldots, l}} \int_{\left(\mathbb{R}^{n}\right)^{m}} \frac{\ell\left(Q_{1, k_{1}}\right)^{\epsilon} \prod_{j=1}^{l}\left|b_{j, k_{j}}\left(y_{j}\right)\right|}{\left(\sum_{j=1}^{m}\left|x-y_{j}\right|\right)^{m n+\epsilon}} \prod_{j=l+1}^{m}\left|g_{j}\left(y_{j}\right)\right| \prod_{j=1}^{m} d y_{j} \\
& +C A \sum_{u=2}^{m} \sum_{k_{2}, \ldots, k_{l}} \sum_{\substack{k_{1}: \ell\left(Q_{1, k_{1}}\right) \leq \ell\left(Q_{i, k_{i}}\right) \\
\text { for all } \\
i=2, \ldots, l}} \int_{\substack{\left.\mathbb{R}^{n}\right)^{m} \\
\text { (n) }}} \frac{\prod_{j=1}^{l}\left|b_{j, k_{j}}\left(y_{j}\right)\right|}{\left(\sum_{j=1}^{m}\left|x-y_{j}\right|\right)^{m n}} \\
& +\phi\left(\frac{\left|y_{1}-y_{u}\right|}{\ell\left(Q_{1, k}\right)}\right) \prod_{j=l+1}^{m}\left|g_{j}\left(y_{j}\right)\right| \prod_{j=1}^{m} d y_{j} \\
& =: T_{1}^{(11)}\left(b_{1}, \ldots, b_{l}, g_{l+1}, \ldots, g_{m}\right)(x)+T_{2}^{(11)}\left(b_{1}, \ldots, b_{l}, g_{l+1}, \ldots, g_{m}\right)(x) \text {. }
\end{aligned}
$$

The assumption $\ell\left(Q_{1, k_{1}}\right) \leq \ell\left(Q_{i, k_{i}}\right), i=2, \ldots, l$, together with property (b) of the Calderón-Zygmund decomposition, imply that

$$
\begin{aligned}
& T_{1}^{(11)}\left(b_{1}, \ldots, b_{l}, g_{l+1}, \ldots, g_{m}\right)(x) \\
& \leq C A \int_{\left(\mathbb{R}^{n}\right)^{m}} \prod_{j=1}^{l}\left(\sum_{k_{j}} \frac{\ell\left(Q_{j, k_{j}}\right)^{\frac{\epsilon}{m}}\left|b_{j, k_{j}}\left(y_{j}\right)\right|}{\left(\ell\left(Q_{j, k_{j}}\right)+\left|x-y_{j}\right|\right)^{n+\frac{\epsilon}{m}}}\right) \\
& \times \prod_{j=l+1}^{m}\left(\frac{\ell\left(Q_{1, k_{1}}\right)^{\frac{\epsilon}{m}}\left|g_{j}\left(y_{j}\right)\right|}{\left(\ell\left(Q_{1, k_{1}}\right)+\left|x-y_{j}\right|\right)^{n+\frac{\epsilon}{m}}}\right) \prod_{j=1}^{m} d y_{j} \\
& \leq C A(\alpha \lambda)^{l / m} \prod_{j=1}^{l} \mathcal{J}_{j, \epsilon / m}(x) \prod_{j=l+1}^{m} \mathcal{M}\left(g_{j}\right)(x),
\end{aligned}
$$

where $\mathcal{M}$ denotes the Hardy-Littlewood maximal operator. On the other hand, we use the fact that $\phi$ is supported in $[-1,1]$, and $\left\|b_{1, k_{1}}\right\|_{L^{1}\left(\mathbb{R}^{n}\right)} \leq C(\alpha \lambda)^{1 / m}\left|Q_{1, k_{1}}\right|$, 
the assumption $\ell\left(Q_{1, k_{1}}\right) \leq \ell\left(Q_{i, k_{i}}\right), i=2, \ldots, l$, to deduce

$$
\begin{aligned}
& T_{2}^{(11)}\left(b_{1}, \ldots, b_{l}, g_{l+1}, \ldots, g_{m}\right)(x) \\
& \leq C A(\alpha \lambda)^{1 / m} \sum_{u=2}^{l} \sum_{k_{1}} \int_{\left(\mathbb{R}^{n}\right)^{m-1}} \prod_{\substack{j=2 \\
j \neq u}}^{l}\left(\sum_{k_{j}} \frac{\ell\left(Q_{j, k_{j}}\right)^{\frac{n}{m-1}}\left|b_{j, k_{j}}\left(y_{j}\right)\right|}{\left(\ell\left(Q_{j, k_{j}}\right)+\left|x-y_{j}\right|\right)^{\frac{m n}{m-1}}}\right) \\
& \times \prod_{j=l+1}^{m}\left(\frac{\ell\left(Q_{1, k_{1}}\right)^{\frac{n}{m-1}}\left|g_{j}\left(y_{j}\right)\right|}{\left(\ell\left(Q_{1, k_{1}}\right)+\left|x-y_{j}\right|\right)^{\frac{m n}{m-1}}}\right) \\
& \times\left(\sum_{k_{u}} \frac{\ell\left(Q_{u, k_{u}}\right)^{\frac{n}{m-1}}\left|b_{u, k_{u}}\left(y_{u}\right)\right|}{\left(\ell\left(Q_{u, k_{u}}\right)+\left|x-y_{u}\right|\right)^{\frac{m n}{m-1}}} \mathbf{1}_{\left(Q_{1, k_{1}}\right)^{*}}\left(y_{u}\right)\right) \prod_{j=2}^{m} d y_{j} \\
& +C A(\alpha \lambda)^{1 / m} \sum_{u=l+1}^{m} \sum_{k_{1}} \int_{\left(\mathbb{R}^{n}\right)^{m-1}} \prod_{j=2}^{l}\left(\sum_{k_{j}} \frac{\ell\left(Q_{j, k_{j}}\right)^{\frac{n}{m-1}}\left|b_{j, k_{j}}\left(y_{j}\right)\right|}{\left(\ell\left(Q_{j, k_{j}}\right)+\left|x-y_{j}\right|\right)^{\frac{m n}{m-1}}}\right) \\
& \times \prod_{\substack{j=l+1 \\
j \neq u}}^{m}\left(\frac{\ell\left(Q_{1, k_{1}}\right)^{\frac{n}{m-1}}\left|g_{j}\left(y_{j}\right)\right|}{\left(\ell\left(Q_{1, k_{1}}\right)+\left|x-y_{j}\right|\right)^{\frac{m n}{m-1}}}\right) \\
& \times\left(\frac{\ell\left(Q_{1, k_{1}}\right)^{\frac{n}{m-1}}}{\left(\ell\left(Q_{1, k_{1}}\right)+\left|x-y_{u}\right|\right)^{\frac{m n}{m-1}}}\left|g_{u}\left(y_{u}\right)\right| \mathbf{1}_{\left(Q_{1, k_{1}}\right) *}\left(y_{u}\right)\right) \prod_{j=2}^{m} d y_{j} \\
& \leq C A(\alpha \lambda)^{l / m} \sum_{u=l+1}^{m} \prod_{j=2}^{l} \mathcal{J}_{j, \frac{n}{m-1}}(x) \prod_{\substack{j=l+1 \\
j \neq u}}^{m} \mathcal{M}\left(g_{j}\right)(x)\left(\mathcal{M}\left(g_{u}\right)(x)+(\alpha \lambda)^{1 / m} \mathcal{J}_{1, \frac{n}{m-1}}(x)\right) .
\end{aligned}
$$

From these estimates, it follows by a standard argument as in the treatment of $E_{\lambda}^{(4)}$ of $m=2$ that

$$
\left|\left\{x \in \mathbb{R}^{n}:\left|T^{(11)}\left(b_{1}, \ldots, b_{l}, g_{l+1}, \ldots, g_{m}\right)(x)\right|>\lambda\right\}\right| \leq C \lambda^{-\frac{1}{m}}\left(\alpha^{-\frac{1}{m}}+A \alpha^{1-\frac{1}{m}}\right) .
$$

For the term $T^{(12)}\left(b_{1}, \ldots, b_{l}, g_{l+1}, \ldots, g_{m}\right)$, we use estimate (2.6), Chebychev's inequality and the $L^{q_{1}}\left(\mathbb{R}^{n}\right) \times \ldots \times L^{q_{m}}\left(\mathbb{R}^{n}\right) \rightarrow L^{q, \infty}\left(\mathbb{R}^{n}\right)$ boundedness of $T$ to deduce the desired estimate. This concludes the case $l=1$. For $l \geq 2$, we decompose $T^{(j)}=T^{(j 1)}+T^{(j 2)}, 2 \leq j \leq l$, as we did for $l=1$ in (2.14). We then prove estimate (2.12) for the terms $T\left(b_{1}, \ldots, b_{l}, g_{l+1}, \ldots, g_{m}\right)$ by suitably adapting the argument as in the case $l=1$ and $m=2$. This argument does not require any new ideas but just some cumbersome notation and for this reason it is omitted. This concludes the proof of Theorem 1.1.

A natural question about Theorem 1.1 is how strong is the imposed assumption (1.8), and what is its relation with the regularity condition (1.4). We will show that, for suitably chosen $A_{t}^{(j)}$, condition (1.8) is actually a consequence of (1.4). This implies that Theorem 1.1 is a strengthening of the analogous theorems in GT2 and $[\mathrm{KS}]$. Precisely, following Proposition 2 of [DM], for any $m>0$, we can construct 
$a_{t}(x, y)$ with the following properties:

$$
\begin{gathered}
a_{t}(x, y)=0, \quad \text { when }|x-y| \geq t^{1 / s}, \\
\int_{\mathbb{R}^{n}} a_{t}(x, y) d x=1
\end{gathered}
$$

for all $y \in \mathbb{R}^{n}, t>0$. This can be achieved by choosing

$$
a_{t}(x, y)=t^{-n / s} \chi_{B\left(y, t^{1 / s}\right)}(x)
$$

where $\chi_{B\left(y, t^{1 / s}\right)}$ denotes the characteristic function on the ball $B\left(y, t^{1 / s}\right)$. Then let $A_{t}$ be the operators which are given by the kernels $a_{t}(x, y)$.

Proposition 2.1. Assume that $T$ has an associated kernel $K\left(x, y_{1}, \cdots, y_{m}\right)$ which satisfies condition (1.4). For each $j=1,2, \cdots, m$, let $\left\{A_{t}^{(j)}\right\}_{t>0}$ be approximations to the identity represented by kernels $a_{t}(x, y)$ satisfying (2.15) and (2.16). Then the kernels $K_{t}^{(j)}(x, y)$ of (1.7) satisfy condition (1.8). More precisely, there exist positive constants $C$ and $\epsilon$ so that

$$
\begin{aligned}
\left|K\left(x, y_{1}, \cdots, y_{j}, \cdots, y_{m}\right)-K_{t}^{(j)}\left(x, y_{1}, \cdots, y_{j}, \cdots, y_{m}\right)\right| & \\
& \leq \frac{A t^{\epsilon / s}}{\left(\left|x-y_{1}\right|+\cdots+\left|x-y_{m}\right|\right)^{m n+\epsilon}}
\end{aligned}
$$

whenever $t^{1 / s} \leq \frac{1}{2}\left|x-y_{j}\right|$.

Proof. For $j=1,2, \cdots, m$, we assume that $t^{1 / s} \leq \frac{1}{2}\left|x-y_{j}\right|$. Then,

$$
\begin{aligned}
\mid K & \left(x, y_{1}, \cdots, y_{j}, \cdots, y_{m}\right)-K_{t}^{(j)}\left(x, y_{1}, \cdots, y_{j}, \cdots, y_{m}\right) \mid \\
= & \left|K\left(x, y_{1}, \cdots, y_{j}, \cdots, y_{m}\right)-\int_{\mathbb{R}^{n}} K\left(x, y_{1}, \cdots, z, \cdots, y_{m}\right) a_{t}\left(z, y_{j}\right) d z\right| \\
= & \mid K\left(x, y_{1}, \cdots, y_{j}, \cdots, y_{m}\right) \int_{\left|z-y_{j}\right|<t^{1 / s}} a_{t}\left(z, y_{j}\right) d z \\
& -\int_{\left|z-y_{j}\right|<t^{1 / s}} K\left(x, y_{1}, \cdots, z, \cdots, y_{m}\right) a_{t}\left(z, y_{j}\right) d z \mid \\
\leq & \int_{\left|z-y_{j}\right|<t^{1 / s}}\left|K\left(x, y_{1}, \cdots, y_{j}, \cdots, y_{m}\right)-K\left(x, y_{1}, \cdots, z, \cdots, y_{m}\right)\right|\left|a_{t}\left(z, y_{j}\right)\right| d z \\
\leq & \frac{A t^{\epsilon / s}}{\left(\left|x-y_{1}\right|+\cdots+\left|x-y_{m}\right|\right)^{m n+\epsilon}} \int_{\left|z-y_{j}\right|<t^{1 / s}}\left|a_{t}\left(z, y_{j}\right)\right| d z \\
\leq & \frac{A t^{\epsilon / s}}{\left(\left|x-y_{1}\right|+\cdots+\left|x-y_{m}\right|\right)^{m n+\epsilon}} .
\end{aligned}
$$

Note that the second equality is obtained by using condition (2.16), and the second inequality follows from (1.4). This completes the proof of Proposition 2.1. 


\section{Multilinear interpolation}

In this section, we will show how to obtain strong type $L^{p_{1}}\left(\mathbb{R}^{n}\right) \times \cdots \times L^{p_{m}}\left(\mathbb{R}^{n}\right) \rightarrow$ $L^{p}\left(\mathbb{R}^{n}\right)$ boundedness results for multilinear operators of generalized CalderónZygmund type starting from a single estimate.

An $m$-linear operator $T: \mathcal{S}\left(\mathbb{R}^{n}\right) \times \cdots \times \mathcal{S}\left(\mathbb{R}^{n}\right) \mapsto \mathcal{S}^{\prime}\left(\mathbb{R}^{n}\right)$ is linear in every entry and consequently it has $m$ formal transposes. The $j$ th transpose $T^{* j}$ of $T$ is defined via

$$
\left\langle T^{* j}\left(f_{1}, \cdots, f_{m}\right), h\right\rangle=\left\langle T\left(f_{1}, \cdots, f_{j-1}, h, f_{j+1}, \cdots, f_{m}\right), f_{j}\right\rangle,
$$

for all $f_{1}, \cdots, f_{m}, g$ in $\mathcal{S}\left(\mathbb{R}^{n}\right)$.

It is easy to check that the kernel $K^{* j}$ of $T^{* j}$ is related to the kernel $K$ of $T$ via the identity

$$
K^{* j}\left(x, y_{1}, \cdots, y_{j-1}, y_{j}, y_{j+1}, \cdots, y_{m}\right)=K\left(y_{j}, y_{1}, \cdots, y_{j-1}, x, y_{j+1}, \cdots, y_{m}\right) .
$$

Note that if a multilinear operator $T$ maps a product of Banach spaces $X_{1} \times \cdots \times X_{m}$ into another Banach space $X$, then the transpose $T^{*, j}$ maps the product of Banach spaces $X_{1} \times \cdots \times X_{j-1} \times X^{*} \times X_{j+1} \times \cdots \times X_{m}$ into $X_{j}^{*}$. Moreover, the norms of $T$ and $T^{*, j}$ are equal. To maintain uniform notation, we may occasionally denote $T$ by $T^{* 0}$ and $K$ by $K^{* 0}$.

Assumption 3.1. Assume that for each $i=1, \cdots, m$ there exist operators $\left\{A_{t}^{(i)}\right\}_{t>0}$ with kernels $a_{t}^{(i)}(x, y)$ that satisfy conditions (1.5) and (1.6) with constants $s$ and $\eta$ and that for every $j=0,1,2, \cdots, m$, there exist kernels $K_{t}^{*, j,(i)}\left(x, y_{1}, \cdots, y_{m}\right)$ such that

$$
\begin{aligned}
& \left\langle T^{* j}\left(f_{1}, \cdots, A_{t}^{(i)} f_{i}, \cdots, f_{m}\right), g\right\rangle \\
& =\int_{\mathbb{R}^{n}} \int_{\left(\mathbb{R}^{n}\right)^{m}} K_{t}^{* j,(i)}\left(x, y_{1}, \cdots, y_{m}\right) f_{1}\left(y_{1}\right) \cdots f_{m}\left(y_{m}\right) g(x) d y_{1} \cdots d y_{m} d x,
\end{aligned}
$$

for all $f_{1}, \cdots, f_{m}$ in $\mathcal{S}\left(\mathbb{R}^{n}\right)$ with $\bigcap_{k=1}^{m} \operatorname{supp} f_{k} \cap \operatorname{supp} g=\emptyset$.

Assumption 3.2. There exist a function $\phi \in C(\mathbb{R})$ with $\operatorname{supp} \phi \subset[-1,1]$ and a constant $\epsilon>0$ so that for every $j=0,1, \ldots, m$ and every $i=1,2, \cdots, m$, we have

$$
\begin{gathered}
\left|K^{* j}\left(x, y_{1}, \cdots, y_{m}\right)-K_{t}^{* j,(i)}\left(x, y_{1}, \cdots, y_{m}\right)\right| \\
\leq \frac{A}{\left(\left|x-y_{1}\right|+\cdots+\left|x-y_{m}\right|\right)^{m n}} \sum_{\substack{k=1 \\
k \neq i}}^{m} \phi\left(\frac{\left|y_{i}-y_{k}\right|}{t^{1 / s}}\right) \\
+\frac{A t^{\epsilon / s}}{\left(\left|x-y_{1}\right|+\cdots+\left|x-y_{m}\right|\right)^{m n+\epsilon}}
\end{gathered}
$$

whenever $t^{1 / s} \leq\left|x-y_{i}\right| / 2$.

Under Assumptions 3.1 and 3.2 we will say that $T$ is an $m$-linear operator with generalized Calderón-Zygmund kernel $K$. The collection of functions $K$ satisfying (3.3) and (3.4) with parameters $m, A, s, \eta$ and $\epsilon$ will be denoted by $m$ $G C Z K(A, s, \eta, \epsilon)$. A kernel $K$ belongs to $m$ - $G C Z K(A, s, \eta, \epsilon)$ exactly when it belongs to $m$ - $G C Z K_{0}(A, s, \eta, \epsilon)$ and all of its adjoints also belong to $m$ - $G C Z K_{0}(A, s, \eta, \epsilon)$. 
We say that $T$ is of class $m-G C Z O(A, s, \eta, \epsilon)$ if $T$ is associated with a kernel $K$ in $m-G C Z K(A, s, \eta, \epsilon)$.

We first have the following:

Theorem 3.1. Assume $T$ is a multilinear operator in $m-G C Z O(A, s, \eta, \epsilon)$. Let $1<q_{1}, q_{2}, \cdots, q_{m}, q<\infty$ be given numbers satisfying

$$
\frac{1}{q_{1}}+\frac{1}{q_{2}}+\cdots+\frac{1}{q_{m}}=\frac{1}{q}
$$

Assume that $T$ maps $L^{q_{1}}\left(\mathbb{R}^{n}\right) \times \cdots \times \times L^{q_{m}}\left(\mathbb{R}^{n}\right)$ to $L^{q}\left(\mathbb{R}^{n}\right)$. Let $p, p_{j}$ be numbers satisfying $1 / m \leq p<\infty, 1 \leq p_{j} \leq \infty$ and $\frac{1}{p}=\frac{1}{p_{1}}+\frac{1}{p_{2}}+\cdots+\frac{1}{p_{m}}$. Then all statements below are valid:

(i) when all $p_{j}>1$, then $T$ can be extended to be a bounded operator from the $m$-fold product $L^{p_{1}}\left(\mathbb{R}^{n}\right) \times \cdots \times L^{p_{m}}\left(\mathbb{R}^{n}\right)$ to $L^{p}\left(\mathbb{R}^{n}\right)$;

(ii) when some $p_{j}=1$, then $T$ can be extended to be a bounded operator from the $m$-fold product $L^{p_{1}}\left(\mathbb{R}^{n}\right) \times \cdots \times L^{p_{m}}\left(\mathbb{R}^{n}\right)$ to $L^{p, \infty}\left(\mathbb{R}^{n}\right)$.

Moreover, there exists a constant $C_{n, m, p_{j}, q_{j}}$ such that the following estimate holds:

$$
\|T\|_{L^{1} \times \cdots \times L^{1} \rightarrow L^{1 / m, \infty}} \leq C_{n, m, p_{j}, q_{j}}\left(A+\|T\|_{L^{q_{1}} \times \cdots \times L^{q_{m}} \rightarrow L^{q}}\right) .
$$

Proof. The proof of Theorem 3.1 is similar to that of Theorem 3, page 140 of [GT2]. We outline the main idea when $m=2$.

Proof of $(i)$. Since $T: L^{q_{1}}\left(\mathbb{R}^{n}\right) \times L^{q_{2}}\left(\mathbb{R}^{n}\right) \rightarrow L^{q}\left(\mathbb{R}^{n}\right)$, it follows that the adjoint with respect to the first variable $T^{* 1}: L^{q^{\prime}}\left(\mathbb{R}^{n}\right) \times L^{q_{2}}\left(\mathbb{R}^{n}\right) \rightarrow L^{q_{1}^{\prime}}\left(\mathbb{R}^{n}\right)$. But then Theorem 1.1 yields that $T^{* 1}: L^{1}\left(\mathbb{R}^{n}\right) \times L^{1}\left(\mathbb{R}^{n}\right) \rightarrow L^{1 / 2, \infty}\left(\mathbb{R}^{n}\right)$. Interpolation (in the complex way) between these estimates yields that $T^{* 1}: L^{s_{1}}\left(\mathbb{R}^{n}\right) \times L^{s_{2}}\left(\mathbb{R}^{n}\right) \rightarrow L^{s, \infty}\left(\mathbb{R}^{n}\right)$ for some $1 / s_{1}+1 / s_{2}=1 / s$, where $s>1$ since $q_{1}^{\prime}>1$. Duality implies that $T: L^{s^{\prime}, \infty}\left(\mathbb{R}^{n}\right) \times L^{s_{2}}\left(\mathbb{R}^{n}\right) \rightarrow L^{s_{1}^{\prime}}\left(\mathbb{R}^{n}\right)$. Theorem 1.1 yields that $T: L^{1}\left(\mathbb{R}^{n}\right) \times$ $L^{1}\left(\mathbb{R}^{n}\right) \rightarrow L^{1 / 2, \infty}\left(\mathbb{R}^{n}\right)$. Interpolating between this estimate and the estimates $T: L^{s^{\prime}, \infty}\left(\mathbb{R}^{n}\right) \times L^{s_{2}}\left(\mathbb{R}^{n}\right) \rightarrow L^{s_{1}^{\prime}}\left(\mathbb{R}^{n}\right)$ and $T: L^{q_{1}}\left(\mathbb{R}^{n}\right) \times L^{q_{2}}\left(\mathbb{R}^{n}\right) \rightarrow L^{q}\left(\mathbb{R}^{n}\right)$ yields boundedness for $T$ in the interior of an open triangle. (Here we use the bilinear Marcinkiewicz interpolation theorem that yields strong-type bounds in the interior of the convex hull of three points at which Lorentz space estimates are known; see GK.) Bootstrapping this argument and using duality and interpolation, we fill in the entire convex region $1<p_{1}, p_{2} \leq \infty$.

Proof of (ii). In the case when some $p_{j}=1$ (say $p_{1}=1$ ), it follows from (i) above that $T: L^{p}\left(\mathbb{R}^{n}\right) \times L^{\infty}\left(\mathbb{R}^{n}\right) \rightarrow L^{p}\left(\mathbb{R}^{n}\right)$ for $1<p<\infty$. Let us prove that $T: L^{1}\left(\mathbb{R}^{n}\right) \times L^{\infty}\left(\mathbb{R}^{n}\right) \rightarrow L^{1, \infty}\left(\mathbb{R}^{n}\right)$. For a fixed function $f_{2} \in L^{\infty}\left(\mathbb{R}^{n}\right)$, we set $\widetilde{K}(x, y)=\int_{\mathbb{R}^{n}} K(x, z, y) f_{2}(y) d y$, and define the 1-linear operator

$$
\begin{aligned}
\widetilde{T}\left(f_{1}\right)(x) & =T\left(f_{1}, f_{2}\right)(x) \\
& =\int_{\mathbb{R}^{n}}\left(\int_{\mathbb{R}^{n}} K(x, z, y) f_{2}(y) d y\right) f_{1}(z) d z \\
& =: \int_{\mathbb{R}^{n}} \widetilde{K}(x, z) f_{1}(z) d z .
\end{aligned}
$$

It follows that the operator $\widetilde{T}$ maps $L^{p}\left(\mathbb{R}^{n}\right)$ to $L^{p}\left(\mathbb{R}^{n}\right)$ with norm $\widetilde{B}=\|\widetilde{T}\|_{L^{p} \rightarrow L^{p}} \leq\|T\|_{L^{p} \times L^{\infty} \rightarrow L^{p}}\left\|f_{2}\right\|_{L^{\infty}} \leq C_{n, 2, p, q_{j}}\left(A+\|T\|_{L^{q_{1}} \times L^{q_{2} \rightarrow L^{q}}}\right)\left\|f_{2}\right\|_{L^{\infty}}$. 
By Assumption 2.2, we have

$$
\begin{aligned}
& \int_{|x-z| \geq 2 t^{1 / s}}\left|\widetilde{K}(x, z)-\widetilde{K}_{t}^{(1)}(x, z)\right| d x \\
& =\int_{|x-z| \geq 2 t^{1 / s}} \mid \int_{\mathbb{R}^{n}} K(x, z, y) f_{2}(y) d y \\
& \quad-\int_{\mathbb{R}^{n}}\left(\int_{\mathbb{R}^{n}} K(x, w, y) f_{2}(y) d y\right) A_{t}(w, z) d w \mid d x \\
& =\int_{|x-z| \geq 2 t^{1 / s}}\left|\int_{\mathbb{R}^{n}}\left(K(x, z, y)-K_{t}^{(2)}(x, z, y)\right) f_{2}(y) d y\right| d x \\
& \leq \int_{|x-z| \geq 2 t^{1 / s}}\left|\int_{\mathbb{R}^{n}} \frac{A}{(|x-z|+|x-y|)^{2 n}} \phi\left(\frac{|z-y|}{t^{1 / s}}\right) f_{2}(y) d y\right| d x \\
& \quad+\int_{|x-z| \geq 2 t^{1 / s}}\left|\int_{\mathbb{R}^{n}} \frac{A t^{\epsilon / s}}{(|x-z|+|x-y|)^{2 n+\epsilon}} f_{2}(y) d y\right| d x \\
& \leq C A\left\|f_{2}\right\|_{L^{\infty}\left(\mathbb{R}^{n}\right) .}
\end{aligned}
$$

Now fix a $\lambda>0$. For every $\alpha>0$, we apply the Calderón-Zygmund decomposition to the function $f_{1}$ at height $\alpha \lambda$ and an argument similar to that of Theorem 1 of DM] to obtain that there exists a constant $C>0$ such that

$$
\begin{aligned}
\left|\left\{x \in \mathbb{R}^{n}:\left|T\left(f_{1}, f_{2}\right)(x)\right|>\lambda\right\}\right| & =\left|\left\{x \in \mathbb{R}^{n}:\left|\widetilde{T} f_{1}(x)\right|>\lambda\right\}\right| \\
& \leq C \lambda^{-1}\left(\widetilde{B}^{p} \alpha^{p\left(1-\frac{1}{p}\right)}+A\left\|f_{2}\right\|_{L^{\infty}\left(\mathbb{R}^{n}\right)}\right)\left\|f_{1}\right\|_{L^{1}\left(\mathbb{R}^{n}\right)} .
\end{aligned}
$$

We now select $\alpha=\left[\left(A+\|T\|_{L^{q_{1}} \times L^{q_{2}} \rightarrow L^{q}}\right)\left\|f_{2}\right\|_{L^{\infty}}\right]^{-1}$. Therefore,

$$
\begin{aligned}
& \left|\left\{x \in \mathbb{R}^{n}:\left|T\left(f_{1}, f_{2}\right)(x)\right|>\lambda\right\}\right| \\
& \quad \leq C \lambda^{-1}\left(A+\|T\|_{L^{q_{1}} \times L^{q_{2}} \rightarrow L^{q}}\right)\left\|f_{1}\right\|_{L^{1}\left(\mathbb{R}^{n}\right)}\left\|f_{2}\right\|_{L^{\infty}\left(\mathbb{R}^{n}\right)},
\end{aligned}
$$

and thus $T: L^{1}\left(\mathbb{R}^{n}\right) \times L^{\infty}\left(\mathbb{R}^{n}\right) \rightarrow L^{1, \infty}\left(\mathbb{R}^{n}\right)$. Since $T: L^{1}\left(\mathbb{R}^{n}\right) \times L^{1}\left(\mathbb{R}^{n}\right) \rightarrow$ $L^{1 / 2, \infty}\left(\mathbb{R}^{n}\right)$, it follows by (linear) interpolation that $T: L^{1}\left(\mathbb{R}^{n}\right) \times L^{p_{2}}\left(\mathbb{R}^{n}\right) \rightarrow$ $L^{p, \infty}\left(\mathbb{R}^{n}\right)$, where $p=p_{2} /\left(p_{2}+1\right)$.

Keeping track of the constants yields (3.5) as the norm of $T$ from $L^{1}\left(\mathbb{R}^{n}\right) \times$ $L^{1}\left(\mathbb{R}^{n}\right)$ to weak $L^{1 / 2}\left(\mathbb{R}^{n}\right)$ is bounded by a multiple of $A$ plus the norm of $T$ on $L^{q_{1}}\left(\mathbb{R}^{n}\right) \times L^{q_{2}}\left(\mathbb{R}^{n}\right)$.

We note that in the proof of our Theorem 1.1, the assumption $q_{1}, q_{2}, \ldots, q_{m}<\infty$ on the indices was necessary (needed for instance in (2.8) in the inequality involving the Marcinkiewicz integral). This restriction presents an inconvenience in certain applications. The main contribution of the result below is to remove this restriction on the indices $q_{j}$.

Theorem 3.2. Assume $T$ is a multilinear operator in $m-G C Z O(A, s, \eta, \epsilon)$. Assume that for some $1 \leq q_{1}, q_{2}, \cdots, q_{m-1} \leq \infty, 1<q_{m}<\infty$ and $0<q<\infty$ with

$$
\frac{1}{q_{1}}+\frac{1}{q_{2}}+\cdots+\frac{1}{q_{m}}=\frac{1}{q}
$$

$T$ maps $L^{q_{1}}\left(\mathbb{R}^{n}\right) \times \cdots \times L^{q_{m}}\left(\mathbb{R}^{n}\right)$ to $L^{q, \infty}\left(\mathbb{R}^{n}\right)$.

(i) Then $T$ can be extended to be a bounded operator from the $m$-fold product $L^{1}\left(\mathbb{R}^{n}\right) \times \cdots \times L^{1}\left(\mathbb{R}^{n}\right)$ to $L^{1 / m, \infty}\left(\mathbb{R}^{n}\right)$. Moreover, for some constant $C_{n, m}$ (that 
depends only on the parameters indicated) we have that

$$
\|T\|_{L^{1} \times \cdots \times L^{1} \rightarrow L^{1 / m, \infty}} \leq C_{n, m}\left(A+\|T\|_{\left.L^{q_{1} \times \cdots \times L^{q m} \rightarrow L^{q, \infty}}\right) .}\right.
$$

(ii) $T$ can be extended to be a bounded operator from the $m$-fold product $L^{p_{1}}\left(\mathbb{R}^{n}\right) \times \cdots \times L^{p_{m}}\left(\mathbb{R}^{n}\right)$ to $L^{1 /\left(m-m_{0}\right), \infty}\left(\mathbb{R}^{n}\right)$, where $p_{i_{k}}=\infty$ for $1 \leq k \leq m_{0}$ and $p_{i_{k}}=1$ for $m_{0}+1 \leq k \leq m$ with norm

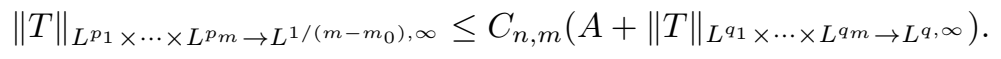

Proof. By Theorem 1.1, it suffices to prove there exist some $1 \leq q_{1}^{*}, q_{2}^{*}, \cdots, q_{m-1}^{*}, q_{m}^{*}$ $<\infty$ and $0<q^{*}<\infty$ with

$$
\frac{1}{q_{1}^{*}}+\frac{1}{q_{2}^{*}}+\cdots+\frac{1}{q_{m}^{*}}=\frac{1}{q^{*}}
$$

such that $T$ maps $L^{q_{1}^{*}}\left(\mathbb{R}^{n}\right) \times \cdots \times L^{q_{m}^{*}}\left(\mathbb{R}^{n}\right)$ to $L^{q^{*}, \infty}\left(\mathbb{R}^{n}\right)$. We outline the main idea when $m=2$. Suppose $q_{1}=\infty$ and $1<q_{2}<\infty$ and suppose $T: L^{q_{1}}\left(\mathbb{R}^{n}\right) \times$ $L^{q_{2}}\left(\mathbb{R}^{n}\right) \rightarrow L^{q_{2}}\left(\mathbb{R}^{n}\right)$. Then $T^{* 1}$ maps $L^{q_{2}^{\prime}}\left(\mathbb{R}^{n}\right) \times L^{q_{2}}\left(\mathbb{R}^{n}\right) \rightarrow L^{1}\left(\mathbb{R}^{n}\right)$. As all the adjoints of $T$ and $T^{* 1}$ have kernels that satisfy assumptions 3.1 and 3.2 it follows from Theorem 3.1 that $T^{* 1}$ maps $L^{p_{1}}\left(\mathbb{R}^{n}\right) \times L^{p_{2}}\left(\mathbb{R}^{n}\right) \rightarrow L^{p}\left(\mathbb{R}^{n}\right)$ for the whole range of exponents. In particular it maps $L^{3}\left(\mathbb{R}^{n}\right) \times L^{3}\left(\mathbb{R}^{n}\right) \rightarrow L^{3 / 2}$. Then so does $T$ and then we can apply Theorem 3.1 again to obtain boundedness for $T$ in the whole range possible.

\section{Applications: Commutators of singular integrals}

In this section we apply Theorem 3.2 to deduce non-trivial bounds for the commutators of A. Calderón. This operator along with its higher counterparts first appeared in the study of the Cauchy integral along Lipschitz curves and in fact these led to the first proof of the $L^{2}$-boundedness of the latter. The first-order commutator is defined as

$$
\mathcal{C}_{2}(a, f)(x)=\int_{\mathbb{R}} \frac{A(x)-A(y)}{(x-y)^{2}} f(y) d y, \text { where } A^{\prime}=a .
$$

It is known that the operator $\mathcal{C}_{2}(a, f)$ extends as a bounded operator from $L^{p}(\mathbb{R}) \times L^{q}(\mathbb{R})$ to $L^{r}(\mathbb{R})$, when $1<p, q \leq \infty$ and $\frac{1}{p}+\frac{1}{q}=\frac{1}{r}, \quad r \neq \infty$. Moreover, if either $p$ or $q$ equals 1 , then $\mathcal{C}_{2}(a, f) \operatorname{maps} L^{p}(\mathbb{R}) \times L^{q}(\mathbb{R})$ to $L^{r, \infty}(\mathbb{R})$ and in particular it maps $L^{1}\left(\mathbb{R}^{n}\right) \times L^{1}(\mathbb{R})$ to $L^{1 / 2, \infty}(\mathbb{R})$. We refer to the articles of $[\mathrm{AC}$, CC] and CM1 for these results.

In this section we obtain all possible Lebesgue strong and weak type bounds concerning the $m$ th-order commutator of Calderón using the techniques developed in the previous sections. We note that the results below for $m \geq 3$ seem to be new (the previous articles only consider the case $m=1$ while CM1 also considers the case $m=2$ ).

We recall the $m$-th commutator given by

$$
\mathcal{C}_{m+1}\left(f, a_{1}, \ldots, a_{m}\right)(x)=\int_{\mathbb{R}} \frac{\prod_{j=1}^{m}\left(A_{j}(x)-A_{j}(y)\right)}{(x-y)^{m}} f(y) d y \quad x \in \mathbb{R},
$$

where $A_{j}^{\prime}=a_{j}$. It is a well-known fact that $f \rightarrow \mathcal{C}_{m+1}\left(f, a_{1}, \ldots, a_{m}\right)$ is a bounded operator on $L^{2}(\mathbb{R})$. Moreover $\mathcal{C}_{m+1}$ can be viewed as an $(m+1)$-linear operator 
which satisfies an $L^{2} \times\left(L^{\infty}\right)^{m} \rightarrow L^{2}$ estimate for each number $m$; that is, there is a constant $C_{m}>0$ such that for all suitable functions $f, a_{1}, \ldots, a_{m}$ we have

$$
\left\|\mathcal{C}_{m+1}\left(f, a_{1}, \ldots, a_{m}\right)\right\|_{L^{2} \times L^{\infty} \times \ldots \times L^{\infty} \rightarrow L^{2}} \leq C_{m}\|f\|_{L^{2}(\mathbb{R})}\left(\prod_{j=1}^{m}\left\|A_{j}^{\prime}\right\|_{L^{\infty}(\mathbb{R})}\right) .
$$

This will be our starting estimate of Theorem 3.2 for the operator $\mathcal{C}_{m+1}$ with $q_{1}=2$, $q_{2}=\cdots=q_{m+1}=\infty$ and $q=2$. There are various estimates for $C_{m}$ ([CJ] and [M]). For example, there is an absolute constant $C>0$ such that $C_{m} \leq C(1+m)^{3}$ for every $m$. See Theorem 3, page 68 of [CJ].

Define

$$
e(x)=\left\{\begin{array}{cc}
1, & x>0 \\
0, & x<0 .
\end{array}\right.
$$

Since $A_{j}^{\prime}=a_{j}$, the $(m+1)$-linear operator $\mathcal{C}_{m+1}\left(f, a_{1}, \ldots, a_{m}\right)$ can be written as

$$
\begin{aligned}
& \mathcal{C}_{m+1}\left(f, a_{1}, \ldots, a_{m}\right)\left(y_{0}\right) \\
& \quad=\int_{\mathbb{R}^{m+1}} K\left(y_{0}, \ldots, y_{m+1}\right) a_{1}\left(y_{1}\right) \ldots a_{m}\left(y_{m}\right) f\left(y_{m+1}\right) d y_{1} \ldots d y_{m+1},
\end{aligned}
$$

where the kernel $K$ is

$$
K\left(y_{0}, \ldots, y_{m+1}\right)=\frac{(-1)^{e\left(y_{m+1}-y_{0}\right) m}}{\left(y_{0}-y_{m+1}\right)^{m+1}} \prod_{\ell=1}^{m} \chi_{\left(\min \left(y_{0}, y_{m+1}\right), \max \left(y_{0}, y_{m+1}\right)\right)}{ }^{\left(y_{\ell}\right) .}
$$

We notice that the adjoint kernels $K^{* j}$ for $j \in\{1, \ldots, m\}$ are

$$
K^{* j}\left(y_{0}, \ldots, y_{m+1}\right)=\frac{(-1)^{e\left(y_{m+1}-y_{j}\right) m}}{\left(y_{j}-y_{m+1}\right)^{m+1}} \prod_{\substack{\ell=0 \\ \ell \neq j}}^{m} \chi_{\left(\min \left(y_{j}, y_{m+1}\right), \max \left(y_{j}, y_{m+1}\right)\right)}\left(y_{\ell}\right),
$$

while $K^{*(m+1)}$ is essentially the same as the original kernel

$K^{*(m+1)}\left(y_{0}, \ldots, y_{m+1}\right)=-\frac{(-1)^{\left(e\left(y_{0}-y_{m+1}\right)+1\right) m}}{\left(y_{0}-y_{m+1}\right)^{m+1}} \prod_{\ell=1}^{m} \chi_{\left(\min \left(y_{0}, y_{m+1}\right), \max \left(y_{0}, y_{m+1}\right)\right.}\left(y_{\ell}\right)$.

We have the following result concerning $\mathcal{C}_{m+1}$.

Theorem 4.1. The mth-order commutator $\mathcal{C}_{m+1}$ in (4.2) is an $(m+1)$-linear operator with a kernel $K$ in $(m+1)-G C Z K(A, 1,1,1)$. Precisely, there exist operators $\left\{A_{t}\right\}_{t>0}$ with kernels $a_{t}(x, y)$ that satisfy conditions (1.5) and (1.6) for all $j \in\{0,1, \ldots, m+1\}$, and for all $i \in\{1, \ldots, m+1\}$ there exist kernels $K_{t}^{* j,(i)}\left(y_{0}, y_{1}, \ldots, y_{m+1}\right)$ such that (1.7) holds and there exists a function $\phi \in C^{1}(\mathbb{R})$ with $\operatorname{supp} \phi \subset[-1,1]$ such that

$\left|K^{* j}\left(y_{0}, y_{1}, \ldots y_{m+1}\right)-K_{t}^{* j,(i)}\left(y_{0}, y_{1}, \ldots, y_{m+1}\right)\right|$

$$
\leq \frac{A}{\left(\sum_{k=1}^{m+1}\left|y_{0}-y_{k}\right|\right)^{m+1}} \sum_{\substack{k=1 \\ k \neq i}}^{m+1} \phi\left(\frac{\left|y_{i}-y_{k}\right|}{t}\right)+\frac{A t}{\left(\sum_{k=1}^{m+1}\left|y_{0}-y_{k}\right|\right)^{m+2}}
$$

whenever $t \leq\left|y_{0}-y_{i}\right| / 2$. 
Proof. We begin by observing that it suffices to prove the required estimate for $j=0$. In fact the case $j=m+1$ is identical to that of $j=0$ while the case $j \in$ $\{1, \ldots, m\}$ is symmetric; one just needs to reverse the roles of $y_{0}$ by that of $y_{j}$. Let us therefore take $j=0$ and consider the following two cases: $(1) i \in\{1,2, \ldots, m\}$ and (2) $i=m+1$.

Let $\phi \in C^{\infty}(\mathbb{R})$ be even, $0 \leq \phi \leq 1, \phi(0)=1$ and supp $(\phi) \subset[-1,1]$. We set $\Phi=\phi^{\prime}$ and $\Phi_{t}(x)=t^{-1} \Phi(x / t)$. Define

$$
A_{t} f(x)=\int_{\mathbb{R}} a_{t}(x, y) f(y) d y, \quad \text { where } a_{t}(x, y)=\Phi_{t}(x-y) \chi_{(x, \infty)}(y) .
$$

Then the kernels $a_{t}(x, y)$ satisfy the conditions (1.5) and (1.6) with constants $s=$ $\eta=1$. For $i \neq 0$ we set

$$
K_{t}^{(i)}\left(y_{0}, \ldots, y_{m+1}\right)=\int_{\mathbb{R}} K\left(y_{0}, \ldots y_{i-1}, z, y_{i+1}, \ldots, y_{m+1}\right) a_{t}\left(z, y_{i}\right) d z .
$$

Case (1). $i \in\{1,2, \ldots, m\}$.

In this case we may take $i=1$ as all the indices in the set $\{1,2, \ldots, m\}$ play the same role. There are two subcases to consider: $y_{0}<y_{m+1}$ and $y_{m+1}<y_{0}$.

Subcase (1.1). $y_{0}<y_{m+1}$.

Here we have that $K\left(y_{0}, y_{1}, \ldots, y_{m+1}\right)-K_{t}^{(1)}\left(y_{0}, y_{1}, \ldots, y_{m+1}\right)$ is equal to

$$
(-1)^{m} \frac{\prod_{k=2}^{m} \chi_{\left(y_{0}, y_{m+1}\right)}\left(y_{k}\right)}{\left(y_{0}-y_{m+1}\right)^{m+1}}\left[\chi_{\left(y_{0}, y_{m+1}\right)}\left(y_{1}\right)-\int_{-\infty}^{y_{1}} \chi_{\left(y_{0}, y_{m+1}\right)}(z) \Phi\left(\frac{z-y_{1}}{t}\right) \frac{d z}{t}\right] .
$$

If the intervals $\left(y_{0}, y_{m+1}\right)$ and $\left(y_{1}-t, y_{1}\right)$ do not intersect, then the difference in (4.5) is zero. If they intersect, since $t<\left|y_{0}-y_{1}\right| / 2$, we must have that $y_{0}<$ $y_{1}-t<y_{1}$. Now if $y_{m+1}>y_{1}$, then the expression inside the square brackets in (4.5) is $1-\int_{y_{1}-t}^{y_{1}} \Phi\left(\frac{z-y_{1}}{t}\right) \frac{d z}{t}$, which is zero. Therefore (4.5) is not zero exactly when $y_{0}<y_{1}-t<y_{m+1}<y_{1}$. In this case we have

$$
\begin{aligned}
& \left|K\left(y_{0}, y_{1}, \ldots, y_{m+1}\right)-K_{t}^{(1)}\left(y_{0}, y_{1}, \ldots, y_{m+1}\right)\right| \\
= & \left|\frac{\prod_{k=2}^{m} \chi_{\left(y_{0}, y_{m+1}\right)}\left(y_{k}\right)}{\left(y_{0}-y_{m+1}\right)^{m+1}} \int_{y_{1}-t}^{y_{m+1}} \Phi\left(\frac{z-y_{1}}{t}\right) \frac{d z}{t}\right| \\
\leq & \frac{C}{\left(\left|y_{0}-y_{1}\right|+\cdots+\left|y_{0}-y_{m+1}\right|\right)^{m+1}} \phi\left(\frac{y_{m+1}-y_{1}}{t}\right) .
\end{aligned}
$$

Subcase (1.2). $y_{m+1}<y_{0}$.

In this case we have that $K\left(y_{0}, y_{1}, \ldots, y_{m+1}\right)-K_{t}^{(1)}\left(y_{0}, y_{1}, \ldots, y_{m+1}\right)$ is equal to

$$
\frac{\prod_{k=2}^{m} \chi_{\left(y_{m+1}, y_{0}\right)}\left(y_{k}\right)}{\left(y_{0}-y_{m+1}\right)^{m+1}}\left[\chi_{\left(y_{m+1}, y_{0}\right)}\left(y_{1}\right)-\int_{-\infty}^{y_{1}} \chi_{\left(y_{m+1}, y_{0}\right)}(z) \Phi\left(\frac{z-y_{1}}{t}\right) \frac{d z}{t}\right] .
$$

If the intervals $\left(y_{m+1}, y_{0}\right)$ and $\left(y_{1}-t, y_{1}\right)$ do not intersect, then (4.6) is zero. If they intersect, then since $t<\left|y_{0}-y_{1}\right| / 2$ we must have $y_{1}-t<y_{1}<y_{0}$. Now if $y_{m+1}<$ $y_{1}-t$ the expression inside the square brackets in (4.6) is $1-\int_{y_{1}-t}^{y_{1}} \Phi\left(\frac{z-y_{1}}{t}\right) \frac{d z}{t}$, 
which is zero. Thus (4.6) is not zero exactly when $y_{1}-t<y_{m+1}<y_{1}<y_{0}$. In this case we have

$$
\begin{aligned}
& \left|K\left(y_{0}, y_{1}, \ldots, y_{m+1}\right)-K_{t}^{(1)}\left(y_{0}, y_{1}, \ldots, y_{m+1}\right)\right| \\
= & \left|\frac{\prod_{k=2}^{m} \chi_{\left(y_{m+1}, y_{0}\right)}\left(y_{k}\right)}{\left(y_{0}-y_{m+1}\right)^{m+1}}\left(1-\int_{y_{m+1}}^{y_{1}} \Phi\left(\frac{z-y_{1}}{t}\right) \frac{d z}{t}\right)\right| \\
\leq & \frac{C}{\left(\left|y_{0}-y_{1}\right|+\cdots+\left|y_{0}-y_{m+1}\right|\right)^{m+1}} \phi\left(\frac{y_{m+1}-y_{1}}{t}\right) .
\end{aligned}
$$

Case (2). $i=m+1$.

Subcase (2.1). $y_{0}<y_{m+1}$.

In this case we have that $K\left(y_{0}, y_{1}, \ldots, y_{m+1}\right)-K_{t}^{(m+1)}\left(y_{0}, y_{1}, \ldots, y_{m+1}\right)$ is equal

$$
(-1)^{m} \frac{\prod_{k=1}^{m} \chi_{\left(y_{0}, y_{m+1}\right)}\left(y_{k}\right)}{\left(y_{0}-y_{m+1}\right)^{m+1}}-\int_{-\infty}^{y_{m+1}}(-1)^{e\left(z-y_{0}\right) m} \frac{\prod_{k=1}^{m} \chi_{\left(y_{0}, z\right)}\left(y_{k}\right)}{\left(y_{0}-z\right)^{m+1}} \Phi\left(\frac{z-y_{m+1}}{t}\right) \frac{d z}{t} .
$$

The first observation is that if $y_{k}<y_{0}$ or $y_{k}>y_{m+1}$ for some $k \in\{1,2, \ldots, m\}$, then the expression in (4.7) is zero. Therefore we must have $y_{0}<y_{k}<y_{m+1}$ for all $k \in\{1,2, \ldots, m\}$ for (4.7) not to be zero. This forces the first product in (4.7) to be equal to 1 . Since $t<\left|y_{0}-y_{m+1}\right| / 2$ in this situation we must have $y_{0}<y_{m+1}-t<y_{m+1}$.

For the integral in (4.7), the range of integration in $z$ is $y_{m+1}-t<z<y_{m+1}$ and $y_{k}<z$ for all $k=1, \ldots, m$. The latter can rephrased as $y_{k_{0}}<z$, where $y_{k_{0}}=\max _{1 \leq k \leq m} y_{k}$. We consider the following two subsubcases:

Subsubcase (2.1.1). $y_{0}<y_{m+1}-t<y_{k_{0}}<y_{m+1}$.

We observe that if $t<\left|y_{0}-y_{m+1}\right| / 2$ and $y_{m+1}-t<z<y_{m+1}$, then $\left|z-y_{0}\right| \geq$ $\left|y_{0}-y_{m+1}\right| / 2$ and also $\left|y_{k_{0}}-y_{0}\right| \geq\left|y_{0}-y_{m+1}\right| / 2$. Since $\Phi=\phi^{\prime}, \phi \in C_{0}^{\infty}(-1,1)$ and $\phi(0)=1$, an integration by parts gives

$$
\begin{aligned}
& \left|K\left(y_{0}, y_{1}, \ldots, y_{m+1}\right)-K_{t}^{(m+1)}\left(y_{0}, y_{1}, \ldots, y_{m+1}\right)\right| \\
& =\left|(-1)^{m} \frac{1}{\left(y_{0}-y_{m+1}\right)^{m+1}}-(-1)^{m} \int_{y_{k_{0}}}^{y_{m+1}} \frac{\prod_{k=1}^{m} \chi_{\left(y_{0}, z\right)}\left(y_{k}\right)}{\left(y_{0}-z\right)^{m+1}} \Phi\left(\frac{z-y_{m+1}}{t}\right) \frac{d z}{t}\right| \\
& =\left|\frac{1}{\left|y_{0}-y_{k_{0}}\right|^{m+1}} \phi\left(\frac{y_{k_{0}}-y_{m+1}}{t}\right)-(m+1) \int_{y_{k_{0}}}^{y_{m+1}} \frac{1}{\left(y_{0}-z\right)^{m+2}} \phi\left(\frac{z-y_{m+1}}{t}\right) d z\right| \\
& \leq \frac{C \phi\left(\frac{y_{k_{0}}-y_{m+1}}{t}\right)}{\left(\left|y_{0}-y_{1}\right|+\cdots+\left|y_{0}-y_{m+1}\right|\right)^{m+1}}+\frac{C t}{\left(\left|y_{0}-y_{1}\right|+\cdots+\left|y_{0}-y_{m+1}\right|\right)^{m+2}} .
\end{aligned}
$$

Subsubcase (2.1.2). $y_{0}<y_{k_{0}}<y_{m+1}-t<y_{m+1}$. 
Here we have

$$
\begin{aligned}
& \left|K\left(y_{0}, \ldots, y_{m+1}\right)-K_{t}^{(m+1)}\left(y_{0}, \ldots, y_{m+1}\right)\right| \\
& \quad=\left|\frac{1}{\left(y_{0}-y_{m+1}\right)^{m+1}}-\int_{y_{m+1}-t}^{y_{m+1}} \frac{1}{\left(y_{0}-z\right)^{m+1}} \Phi\left(\frac{z-y_{m+1}}{t}\right) \frac{d z}{t}\right| \\
& \quad=(m+1)\left|\int_{y_{m+1}-t}^{y_{m+1}} \frac{1}{\left(y_{0}-z\right)^{m+2}} \phi\left(\frac{z-y_{m+1}}{t}\right) d z\right| \\
& \quad \leq \frac{C t}{\left(\left|y_{0}-y_{m+1}\right|\right)^{m+2}} \leq \frac{C^{\prime} t}{\left(\left|y_{0}-y_{1}\right|+\cdots+\left|y_{0}-y_{m+1}\right|\right)^{m+2}} .
\end{aligned}
$$

Subcase (2.2). $y_{m+1}<y_{0}$.

We have $K\left(y_{0}, y_{1}, \ldots, y_{m+1}\right)-K_{t}^{(m+1)}\left(y_{0}, y_{1}, \ldots, y_{m+1}\right)$ is equal to (4.8)

$$
\frac{\prod_{k=1}^{m} \chi_{\left(y_{m+1}, y_{0}\right)}\left(y_{k}\right)}{\left(y_{0}-y_{m+1}\right)^{m+1}}-\int_{-\infty}^{y_{m+1}}(-1)^{e\left(z-y_{0}\right) m} \frac{\prod_{k=1}^{m} \chi_{\left(z, y_{0}\right)}\left(y_{k}\right)}{\left(y_{0}-z\right)^{m+1}} \Phi\left(\frac{z-y_{m+1}}{t}\right) \frac{d z}{t} .
$$

In this case we have $y_{m+1}-t<y_{m+1}<y_{0}$. We first observe that (4.8) is zero unless all $y_{k}$ lie in the interval $\left(y_{m+1}-t, y_{0}\right)$.

The range of integration for $z$ is the intersection of the two intervals $\left(y_{m+1}-\right.$ $\left.t, y_{m+1}\right)$ and $\left(-\infty, y_{k_{1}}\right)$, where $y_{k_{1}}=\min _{1 \leq k \leq m} y_{k}$. We consider the following two subsubcases:

Subsubcase (2.2.1). $y_{m+1}-t<y_{m+1}<y_{k_{1}}<y_{0}$.

In this case all the $y_{k}$ are between $y_{m+1}$ and $y_{0}$ and we have

$$
\begin{aligned}
& \left|K\left(y_{0}, \ldots, y_{m+1}\right)-K_{t}^{(m+1)}\left(y_{0}, \ldots, y_{m+1}\right)\right| \\
& \quad=\left|\frac{1}{\left(y_{0}-y_{m+1}\right)^{m+1}}-\int_{y_{m+1}-t}^{y_{m+1}} \frac{1}{\left(y_{0}-z\right)^{m+1}} \Phi\left(\frac{z-y_{m+1}}{t}\right) \frac{d z}{t}\right| \\
& \quad=(m+1)\left|\int_{y_{m+1}-t}^{y_{m+1}} \frac{1}{\left(y_{0}-z\right)^{m+2}} \phi\left(\frac{z-y_{m+1}}{t}\right) d z\right| \\
& \quad \leq \frac{C t}{\left(\left|y_{0}-y_{m+1}\right|\right)^{m+2}} \leq \frac{C^{\prime} t}{\left(\left|y_{0}-y_{1}\right|+\cdots+\left|y_{0}-y_{m+1}\right|\right)^{m+2}}
\end{aligned}
$$

since $\left|z-y_{0}\right| \geq\left|y_{0}-y_{m+1}\right|$ in this case.

Subsubcase (2.2.2). $y_{m+1}-t<y_{k_{1}}<y_{m+1}<y_{0}$.

Here we apply an integration by parts as we did previously to obtain the estimate

$$
\begin{aligned}
& \left|K\left(y_{0}, \ldots, y_{m+1}\right)-K_{t}^{(m+1)}\left(y_{0}, \ldots, y_{m+1}\right)\right| \\
& \quad=\left|\int_{y_{m+1}-t}^{y_{k_{1}}} \frac{1}{\left(y_{0}-z\right)^{m+1}} \Phi\left(\frac{z-y_{m+1}}{t}\right) \frac{d z}{t}\right| \\
& \quad \leq \frac{C}{\left|y_{0}-y_{k_{1}}\right|^{m+1}} \phi\left(\frac{y_{k_{1}}-y_{m+1}}{t}\right)+\frac{(m+1) t}{\left(\left|y_{0}-y_{k_{1}}\right|\right)^{m+2}} \\
& \quad \leq \frac{C \phi\left(\frac{y_{k_{1}}-y_{m+1}}{t}\right)}{\left(\left|y_{0}-y_{1}\right|+\cdots+\left|y_{0}-y_{m+1}\right|\right)^{m+1}}+\frac{t}{\left(\left|y_{0}-y_{1}\right|+\cdots+\left|y_{0}-y_{m+1}\right|\right)^{m+2}}
\end{aligned}
$$

since in this case we have $\left|y_{0}-y_{k_{1}}\right| \geq\left|y_{0}-y_{k}\right|$ for all $k=1,2, \ldots, m+1$.

The proof of the theorem is complete. 
Applying Theorem 3.2 we deduce the following result concerning the $m$ th-order commutators. This contains several new endpoint estimates for them.

Corollary 4.2. Let $0<1 / p=\sum_{j=1}^{m+1} 1 / p_{j}$. Then the mth-order commutator $\mathcal{C}_{m}$ maps $L^{p_{1}}(\mathbb{R}) \times \cdots \times L^{p_{m+1}}(\mathbb{R})$ to $L^{p, \infty}(\mathbb{R})$ whenever $1 \leq p_{1}, \ldots, p_{m+1} \leq \infty$ and it also maps $L^{p_{1}}(\mathbb{R}) \times \cdots \times L^{p_{m+1}}(\mathbb{R})$ to $L^{p}(\mathbb{R})$ when $1<p_{j}<\infty$ for all $j$. In particular it maps $L^{1}(\mathbb{R}) \times \cdots \times L^{1}(\mathbb{R})$ to $L^{1 /(m+1), \infty}(\mathbb{R})$.

\section{The HigheR-Dimensional COMmUtator}

In this section we obtain new bounds for the higher-dimensional commutator introduced by Christ and Journé C.J. We discuss a connection between this commutator and the bilinear Hilbert transforms and we use this connection to obtain some non-trivial new off-diagonal estimates for the former. The results of this section are inspired by Calderón's original idea to express the 1-dimensional commutators in terms of the bilinear Hilbert transforms.

The higher-dimensional commutator is defined as

$$
\mathcal{C}_{2}^{(n)}(f, a)(x)=\text { p.v. } \int_{\mathbb{R}^{n}} K(x-y) \int_{0}^{1} f(y) a((1-t) x+t y) d t d y,
$$

where $K(x)$ is a Calderón-Zygmund kernel in dimension $n$ and $f, a$ are functions on $\mathbb{R}^{n}$. Christ and Journé [CJ] proved that $\mathcal{C}_{2}^{(n)}$ is bounded from $L^{p}\left(\mathbb{R}^{n}\right) \times L^{\infty}\left(\mathbb{R}^{n}\right)$ to $L^{p}\left(\mathbb{R}^{n}\right)$ for $1<p<\infty$. Here we discuss some off-diagonal bounds $L^{p} \times L^{q} \rightarrow L^{r}$, whenever $1 / p+1 / q=1 / r$ and $1<p, q, r<\infty$.

To obtain these bounds we will exploit a connection with the bilinear Hilbert transform defined as

$$
H_{\alpha, \beta}(f, g)(x)=\text { p.v. } \int_{-\infty}^{+\infty} f(x-\alpha t) g(x-\beta t) \frac{d t}{t},
$$

where $f, g$ are functions on the line and $x, \alpha, \beta \in \mathbb{R}$. This operator was first shown to be bounded by Lacey and Thiele [LT1, [LT2] in the range

$$
1<p, q \leq \infty, \quad 2 / 3<r<\infty, \quad 1 / p+1 / q=1 / r .
$$

Uniform $L^{r}$ bounds (in $\alpha, \beta$ ) for it were obtained by Grafakos and Li GL in the local $L^{2}$ case, (i.e., the case when $2<p, q, r^{\prime}<\infty$ ) and extended by $\mathrm{Li}[\mathrm{L}]$ in the hexagonal region

$$
1<p, q, r<\infty, \quad\left|\frac{1}{p}-\frac{1}{q}\right|<\frac{1}{2}, \quad\left|\frac{1}{p}-\frac{1}{r^{\prime}}\right|<\frac{1}{2}, \quad\left|\frac{1}{q}-\frac{1}{r^{\prime}}\right|<\frac{1}{2} .
$$

As the operator $\mathcal{C}_{2}^{(n)}(f, a)$ is $n$-dimensional, we will need to "transfer" $H_{\alpha, \beta}$ in higher dimensions. To achieve this we use rotations. We have the following lemma:

Lemma 5.1. Suppose that $K$ is kernel in $\mathbb{R}^{2 n}$ (which may be a distribution) and let $T_{K}$ be the bilinear singular integral operator associated with $K$

$$
T_{K}(f, g)(x)=\iint K(x-y, x-z) f(y) g(z) d y d z .
$$

Assume that $T_{K}$ is bounded from $L^{p}\left(\mathbb{R}^{n}\right) \times L^{q}\left(\mathbb{R}^{n}\right) \rightarrow L^{r}\left(\mathbb{R}^{n}\right)$ with norm $\|T\|$ when $1 / p+1 / q=1 / r$. Let $M$ be an $n \times n$ invertible matrix. Define $a 2 n \times 2 n$ invertible 
matrix $\widetilde{M}=\left(\begin{array}{cc}M & O \\ O & M\end{array}\right)$, where $O$ is the zero $n \times n$ matrix. Then the operator $T_{K \circ \widetilde{M}}$ is also bounded from $L^{p}\left(\mathbb{R}^{n}\right) \times L^{q}\left(\mathbb{R}^{n}\right) \rightarrow L^{r}\left(\mathbb{R}^{n}\right)$ with norm at most $\|T\|$.

Proof. To prove the lemma we note that

$$
T_{K \circ \widetilde{M}}(f, g)(x)=T_{K}\left(f \circ M^{-1}, g \circ M^{-1}\right)(M x),
$$

from which it follows that

$$
\begin{aligned}
\left\|T_{K \circ \widetilde{M}}(f, g)\right\|_{L^{r}} & =(\operatorname{det} M)^{-1 / r}\left\|T_{K}\left(f \circ M^{-1}, g \circ M^{-1}\right)\right\|_{L^{r}} \\
& \leq(\operatorname{det} H)^{-1 / r}\|T\|\left\|f \circ M^{-1}\right\|_{L^{p}}\left\|g \circ M^{-1}\right\|_{L^{q}} \\
& =\|T\|(\operatorname{det} M)^{-1 / r}\|T\|\|f\|_{L^{p}}(\operatorname{det} M)^{1 / p}\|g\|_{L^{q}}(\operatorname{det} M)^{1 / p} \\
& =\|T\| .
\end{aligned}
$$

We apply Lemma 5.1 to the bilinear Hilbert transform. Let $e_{1}=(1,0, \ldots, 0)$ be the standard coordinate vector on $\mathbb{R}^{n}$. We begin with the observation that the operator

$$
\mathcal{H}_{\alpha, \beta}^{e_{1}}(f, g)(x)=\text { p.v. } \int_{-\infty}^{+\infty} f\left(x-\alpha t e_{1}\right) g\left(x-\beta t e_{1}\right) \frac{d t}{t}, \quad x \in \mathbb{R}^{n}
$$

defined for functions $f, g$ on $\mathbb{R}^{n}$ is bounded from $L^{p}\left(\mathbb{R}^{n}\right) \times L^{q}\left(\mathbb{R}^{n}\right) \rightarrow L^{r}\left(\mathbb{R}^{n}\right)$ for the same range of indices as the bilinear Hilbert transform. Indeed, the operator $\mathcal{H}_{\alpha, \beta}^{e_{1}}$ can be viewed as the classical one-dimensional bilinear Hilbert transform in the coordinate $x_{1}$ followed by the identity operator in the remaining coordinates $x_{2}, \ldots, x_{n}$, where $x=\left(x_{1}, \ldots, x_{n}\right)$. By Lemma 5.1 for an invertible $n \times n$ matrix $M$ we have that

$\mathcal{H}_{\alpha, \beta}^{e_{1}}\left(f \circ M^{-1}, g \circ M^{-1}\right)(M x)=$ p.v. $\int_{-\infty}^{+\infty} f\left(x-\alpha t M^{-1} e_{1}\right) g\left(x-\beta t M^{-1} e_{1}\right) \frac{d t}{t}, x \in \mathbb{R}^{n}$

maps $L^{p}\left(\mathbb{R}^{n}\right) \times L^{q}\left(\mathbb{R}^{n}\right) \rightarrow L^{r}\left(\mathbb{R}^{n}\right)$ with norm the same as the one-dimensional bilinear Hilbert transform $H_{\alpha, \beta}$ whenever the indices $p, q, r$ satisfy (5.2). If $M$ is a rotation (i.e. an orthogonal matrix), then $M^{-1} e_{1}$ can be any unit vector in $\mathbb{S}^{n-1}$. We conclude that the family of operators

$$
\mathcal{H}_{\alpha, \beta}^{\theta}(f, g)(x)=\text { p.v. } \int_{-\infty}^{+\infty} f(x-\alpha t \theta) g(x-\beta t \theta) \frac{d t}{t}, \quad x \in \mathbb{R}^{n}
$$

is bounded from $L^{p}\left(\mathbb{R}^{n}\right) \times L^{q}\left(\mathbb{R}^{n}\right)$ to $L^{r}\left(\mathbb{R}^{n}\right)$ with a bound independent of $\theta \in \mathbb{S}^{n-1}$ whenever the indices $p, q, r$ satisfy (5.2). This bound is also independent of $\alpha, \beta$ whenever the indices $p, q, r$ satisfy (5.3).

It remains to express the higher-dimensional commutator $\mathcal{C}_{2}^{(n)}$ in terms of the operators $\mathcal{H}_{\alpha, \beta}^{\theta}$. Here we make the assumption that $K$ is an odd homogeneous singular integral operator on $\mathbb{R}^{n}$, such as a Riesz transform. For a fixed $x \in \mathbb{R}^{n}$ we apply polar coordinates centered at $x$ by writing $y=x-r \theta$. Then we can express the higher-dimensional commutator in (5.1) as

$$
\int_{\mathbb{S}^{n-1}} \int_{0}^{\infty} \frac{K(\theta)}{r^{n}} \int_{0}^{1} f(x-r \theta) a(x-t r \theta) d t r^{n-1} d r d \theta .
$$


Changing variables from $\theta \rightarrow-\theta, r \rightarrow-r$ and using that $K(\theta)$ is odd we write this expression as

$$
\int_{\mathbb{S}^{n-1}} \int_{-\infty}^{0} K(\theta) \int_{0}^{1} f(x-r \theta) a(x-\operatorname{tr} \theta) d t \frac{d r}{r} d \theta .
$$

Averaging the (5.4) and (5.5) we arrive at the identity

$$
\mathcal{C}_{2}^{(n)}(f, a)(x)=\frac{1}{2} \int_{\mathbb{S}^{n-1}} K(\theta) \int_{0}^{1} \mathcal{H}_{1, t}^{\theta}(f, a)(x) d t d \theta .
$$

This certainly implies the boundedness of $\mathcal{C}_{2}^{(n)}$ from $L^{p}\left(\mathbb{R}^{n}\right) \times L^{q}\left(\mathbb{R}^{n}\right)$ to $L^{r}\left(\mathbb{R}^{n}\right)$ whenever the indices $p, q, r$ satisfy (5.3). Interpolation with the known $L^{p} \times L^{\infty} \rightarrow$ $L^{p}$ bounds yield the following:

Corollary 5.2. Let $K$ be an odd homogeneous singular integral on $\mathbb{R}^{n}$. Then the $n$ dimensional commutator $\mathcal{C}_{2}^{(n)}$ associated with $K$ maps $L^{p}\left(\mathbb{R}^{n}\right) \times L^{q}\left(\mathbb{R}^{n}\right) \rightarrow L^{r}\left(\mathbb{R}^{n}\right)$ whenever $1 / p+1 / q=1 / r$ and $(1 / p, 1 / q, 1 / r)$ lies in the open convex hull of the pentagon with vertices $(0,1 / 2,1 / 2),(0,0,0),(1,0,1),(1 / 2,1 / 2,1)$, and $(1 / 6,4 / 6,5 / 6)$.

Bounds for $\mathcal{C}_{2}^{(n)}$ near the vertex $(0,1,1)$ and on $L^{r}\left(\mathbb{R}^{n}\right)$ for $r \leq 1$ (in particular, the $L^{1} \times L^{1} \rightarrow L^{1 / 2, \infty}$ estimate derived in the 1-dimensional case) remain elusive at the moment.

\section{ACKNOWLEDGMENTS}

The authors would like to thank Dachun Yang for his comments. The third author would also like to thank Steve Hofmann for helpful discussions and suggestions, and he thanks the Department of Mathematics of the University of MissouriColumbia for its hospitality.

\section{REFERENCES}

[AC] A.P. Calderón, Commutators of singular integrals, Proc. Nat. Acad. Sci. U.S.A., 53 (1965), 1092-1099. MR0177312 (31:1575)

[CC] C.P. Calderón, On commutators of singular integrals, Studia Math., 53 (1975), 139-174. MR0380518 (52:1418)

[CJ] M. Christ and J.-L. Journé, Polynomial growth estimates for multilinear singular integral operators, Acta Math., 159 (1987), 51-80. MR906525 (89a:42024)

[CM1] R. Coifman and Y. Meyer, On commutators of singular integral and bilinear singular integrals, Trans. Amer. Math. Soc., 212 (1975), 315-331. MR0380244 (52:1144)

[CM2] R. Coifman and Y. Meyer, Au delà des opérateurs pseudo-différentiels, Astérisque, $\mathbf{5 7}$ (1978). MR518170 (81b:47061)

[CM3] R. Coifman and Y. Meyer, Ondelettes et opérateurs, III, Hermann, Paris, 1990. MR.1160989 (93i:42004)

[DM] X.T. Duong and A. McIntosh, Singular integral operators with non-smooth kernels on irregular domains, Rev. Mat. Iberoamericana, 15 (1999), 233-265. MR 1715407 (2001e:42017a)

[G] L. Grafakos, Classical and Modern Fourier Analysis, Pearson Education, Inc., Upper Saddle River, New Jersey, 2004. MR2449250

[GK] L. Grafakos and N. Kalton, Some remarks on multilinear maps and interpolation, Math. Ann., 319 (2001), 151-180. MR.1812822 (2002a:46032)

[GL] L. Grafakos and X. Li, Uniform bounds for the bilinear Hilbert transforms, I, Ann. of Math. (2), 159 (2004), 889-933. MR2113017(2006e:42011) 
[GT1] L. Grafakos and R.H. Torres, On multilinear singular integrals of Calderón-Zygmund type, Proceedings of the 6th International Conference on Harmonic Analysis and Partial Differential Equations (El Escorial, 2000). Publ. Mat. (2002), Extra, 57-91. MR1964816 (2004c:42031)

[GT2] L. Grafakos and R.H. Torres, Multilinear Calderón-Zygmund theory, Adv. in Math., 165 (2002), 124-164. MR.1880324(2002j:42029)

[FS] C. Fefferman and E.M. Stein, Some maximal inequalities, Amer. J. Math., 93 (1971), 107-115. MR0284802(44:2026)

[KS] C. E. Kenig and E.M. Stein, Multilinear estimates and fractional integration, Math. Res. Letters, 6 (1999), 1-15. MR1682725 (2000k:42023a)

[LT1] M. Lacey and C. Thiele, $L^{p}$ estimates on the bilinear Hilbert transform for $2<p<\infty$, Ann. of Math. (2), 146 (1997), 693-724. MR.1491450 (99b:42014)

[LT2] M. Lacey and C. Thiele, On Calderón's conjecture, Ann. of Math. (2), 149 (1999), 475496. MR.1689336 (2000d:42003)

[L] X. Li, Uniform bounds for the bilinear Hilbert transforms, II, Rev. Mat. Iberoamericana, 22 (2006), 1069-1126. MR.2320411 (2008c:42014)

[M] T. Murai, A real variable method for the Cauchy transforms, and analytic capacity, Lecture Notes in Math., 1307, Springer, Berlin, 1988. MR.944308 (89k:30022)

[St] E.M. Stein, Harmonic Analysis: Real variable methods, orthogonality and oscillatory integrals, Princeton Univ. Press, 1993. MR.1232192 (95c:42002)

Department of Mathematics, Macquarie University, NSW, 2109, Australia

E-mail address: duong@ics.mq.edu.au

Department of Mathematics, University of Missouri, Columbia, Missouri 65211

E-mail address: loukas@math.missouri.edu

Department of Mathematics, Zhongshan University, Guangzhou, 510275, People's RePUBLIC OF CHINA

E-mail address: mcsylx@mail.sysu.edu.cn 